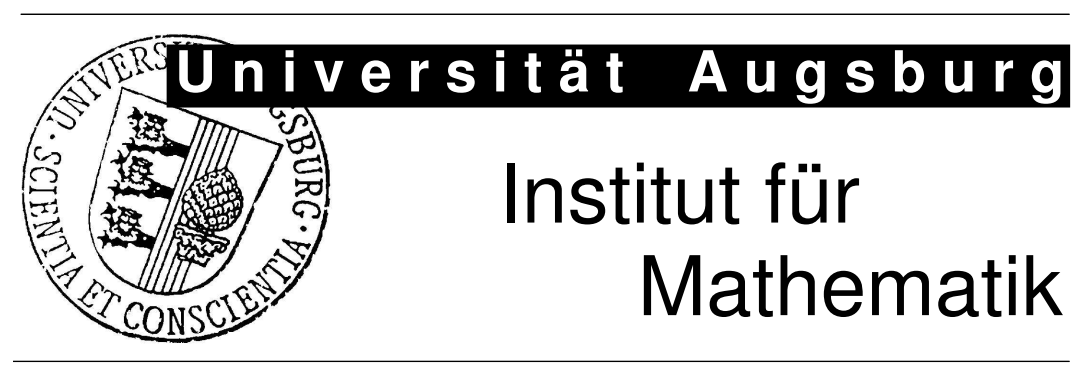

Tatjana Stykel, Alexander Vasilyev

A two-step Model Reduction Approach for Mechanical Systems With Moving Loads 


\section{Impressum:}

\section{Herausgeber:}

Institut für Mathematik

Universität Augsburg

86135 Augsburg

http://www . math. uni-augsburg.de/de/forschung/preprints.html

\section{ViSdP:}

Tatjana Stykel

Institut für Mathematik

Universität Augsburg

86135 Augsburg

Preprint: Sämtliche Rechte verbleiben den Autoren (C) 2015 


\title{
A TWO-STEP MODEL REDUCTION APPROACH FOR MECHANICAL SYSTEMS WITH MOVING LOADS *
}

\author{
TATJANA STYKEL ${ }^{\dagger}$ AND ALEXANDER VASILYEV ${ }^{\ddagger}$
}

\begin{abstract}
We consider model order reduction of mechanical systems with moving loads. Such systems have a time-varying input matrix that makes it difficult the direct application of standard model reduction methods. In this paper, we present a two-step model reduction approach for systems with moving loads which is based on a low-rank approximation of the input matrix and applying Krylov subspace methods to the resulting linear time-invariant system with a modified input. Numerical results demonstrate the properties of the proposed model reduction method.
\end{abstract}

Key words. mechanical systems, moving loads, model reduction

1. Introduction. In structural dynamics, the moving load problem has received a lot of attention because of its importance in many practical applications. Mechanical systems with moving loads arise, for example, in modelling of bridges with moving vehicles [17, 32], cableways [17], cranes [42] or working gears [39]. One of the most popular methods for simulation of the dynamic behaviour of such systems is a finite element method (FEM) based on a variational formulation of the structural mechanics problem, e.g., [41]. In the engineering literature, the principle of virtual work is used to derive the FEM approximations, see [5]. The FEM discretization of a system subjected to moving loads yields a linear time-varying (LTV) second-order system

$$
\begin{aligned}
M \ddot{q}(t)+D \dot{q}(t)+K q(t) & =B(t) u(t), \\
y(t) & =C(t) q(t),
\end{aligned}
$$

where $M, D, K \in \mathbb{R}^{N \times N}$ are the mass, damping and stiffness matrices, respectively, $q(t) \in \mathbb{R}^{N}$ is an unknown vector of generalized coordinates, $u(t) \in \mathbb{R}^{m}$ is the input, and $y(t) \in \mathbb{R}^{p}$ is an output describing a response in a domain of interest. Furthermore, $B(t) \in \mathbb{R}^{N \times m}$ and $C(t) \in \mathbb{R}^{p \times N}$ are the time-dependent input and output matrices describing, respectively, force and observation positions at time $t \in[0, T]$.

As an example, we consider a simply supported beam excited by a moving force. The vibration of the beam is modelled by an Euler-Bernoulli equation

$$
\mu A \frac{\partial^{2}}{\partial t^{2}} w(x, t)+2 \mu A \omega_{\mathrm{d}} \frac{\partial}{\partial t} w(x, t)+E J \frac{\partial^{4}}{\partial x^{4}} w(x, t)=f(x, t)
$$

with the initial and boundary conditions

$$
\begin{gathered}
w(x, 0)=0, \quad \frac{\partial}{\partial t} w(x, 0)=0, \\
w(0, t)=0, \quad \frac{\partial^{2}}{\partial x^{2}} w(0, t)=0, \quad w(l, t)=0, \quad \frac{\partial^{2}}{\partial x^{2}} w(l, t)=0,
\end{gathered}
$$

*This work was supported by the DFG project "Model reduction for elastic multibody systems with moving interactions", grant STY 58/1-1.

${ }^{\dagger}$ Institut für Mathematik, Universität Augsburg, Universitätsstr. 14, 86159 Augsburg, Germany (stykel@math.uni-augsburg.de).

$\ddagger$ Institut für Mathematik, Universität Augsburg, Universitätsstr. 14, 86159 Augsburg, Germany (alexander.vasilyev@math.uni-augsburg.de). 
where $w(x, t)$ is a vertical displacement of the beam, $\mu$ is the mass density, $A$ is the cross-sectional area, $E$ is the Young modulus, $J$ is the moment of inertia of the crosssection, $\omega_{\mathrm{d}}$ is the circular frequency of damping, and $l$ is the length of the beam. The external force acting on the beam is described by a function

$$
f(x, t)=\sum_{j=1}^{m} \varrho_{j}(x, t) u_{j}(t)
$$

where $\varrho_{j}(x, t)$ and $u_{j}(t)$ are the normalized distribution and magnitude of the $j$-th excitation, respectively. The time dependence in $\varrho_{j}(x, t)$ indicates a time-varying excitation or a moving load. Applying the FEM to (1.2), we get system (1.1) with the symmetric, positive definite matrices $M, D$ and $K$. The entries of the input matrix $B(t)=\left[b_{i j}(t)\right]$ have the form

$$
b_{i j}(t)=\int_{0}^{l} \varrho_{j}(x, t) \varphi_{N, i}(x) d x, \quad i=1, \ldots, N, j=1, \ldots, m,
$$

where $\varphi_{N, i}(x)$ are the FEM basis functions and $N$ is the number of degrees of freedom. If the beam is excited by the point forces, then

$$
\varrho_{j}(x, t)=\delta\left(x-\xi_{j}(t)\right),
$$

where $\delta(x)$ is the Dirac delta function and $\xi_{j}(t)$ is an instantaneous position of the $j$-th force at time $t$. In this case, the entries of $B(t)$ are defined especially simply as

$$
b_{i j}(t)=\varphi_{N, i}\left(\xi_{j}(t)\right)
$$

Unfortunately, simulation of complex mechanical structures requires frequently an overwhelming computational effort due to a large number of degrees of freedom. In order to reduce the computational complexity when solving the large-scale system (1.1) numerically, we may use model order reduction. It consists of approximating (1.1) by a reduced model

$$
\begin{aligned}
\widetilde{M} \ddot{\widetilde{q}}(t)+\widetilde{D} \dot{\widetilde{q}}(t)+\widetilde{K} \widetilde{q}(t) & =\widetilde{B}(t) u(t), \\
\widetilde{y}(t) & =\widetilde{C}(t) \widetilde{q}(t),
\end{aligned}
$$

where $\widetilde{M}, \widetilde{D}, \widetilde{K} \in \mathbb{R}^{r \times r}, \widetilde{B}(t) \in \mathbb{R}^{r \times m}, \widetilde{C}(t) \in \mathbb{R}^{p \times r}$ and $r \ll N$. Such a model can be computed by projection

$$
\begin{gathered}
\widetilde{M}=W^{T} M V, \quad \widetilde{D}=W^{T} D V, \quad \widetilde{K}=W^{T} K V, \\
\widetilde{B}(t)=W^{T} B(t), \quad \widetilde{C}(t)=C(t) V,
\end{gathered}
$$

where the projection matrices $V, W \in \mathbb{R}^{N \times r}$ are chosen such that the approximation error $\widetilde{y}-y$ is small in an appropriate norm. For linear time-invariant (LTI) secondorder systems, the projection matrices can be determined using balanced truncation $[8,25,31]$ or (rational) Krylov subspace techniques [2, 3, 33]. The balanced truncation method has been extended to LTV systems in $[34,36]$. It relies on solving two Lyapunov differential equations and is computationally expensive and memory demanding. Another model reduction approach for LTV systems has been presented in $[20,29]$. It is based on converting the LTV system to a LTI system by time discretization and applying the recycled Krylov subspace technique. Note that these 
both model reduction approaches are applicable to general LTV systems, where all system matrices are time-varying. In system (1.1), however, the state matrices $M$, $D$ and $K$ are time-independent, and only the input and output matrices depend on time. Such a system can be reformulated as the LTI system

$$
\begin{aligned}
M \ddot{q}(t)+D \dot{q}(t)+K q(t) & =u_{\text {new }}(t), \\
y_{\text {new }}(t) & =q(t)
\end{aligned}
$$

with the input $u_{\text {new }}(t)=B(t) u(t) \in \mathbb{R}^{N}$ and the output $y_{\text {new }}(t) \in \mathbb{R}^{N}$ of large dimension. Model reduction of systems with many inputs and outputs has been considered in $[10,13,24]$. The methods proposed there are based on a low-rank approximation of the input and output matrices using a singular value decomposition combined with standard model reduction techniques. Unfortunately, these methods cannot be applied to system (1.5), since the input and output matrices in (1.5) are the identity matrices. In [2, 19], an $\mathcal{H}_{2}$-optimal model reduction method has been developed which is based on an iterative rational Krylov algorithm (IRKA). This method can also be applied to systems with many inputs and outputs. It should, however, be noticed that for such systems, IRKA may exhibit a slow convergence or even the iteration may stagnate.

Model reduction of systems with moving loads has been considered in [14, 15, 22], where the variability of the external force was described either by switched systems or by parameter-dependent systems. In the latter case, parametric model reduction algorithms were examined in the context of the moving load problem. In this paper, we consider the modelling of moving loads by a time-varying input matrix and present a two-step model reduction method for the resulting LTV system. Our approach is based on a transformation of the LTV system (1.1) into a LTI system by approximating the input matrix and application of structure-preserving Krylov subspace model reduction methods.

The paper is organized as follows. In Section 2, we consider a low-rank approximation of the time-varying input matrix $B(t)$ using interpolation and a linear least-squares method with two different types of ansatz functions. We also derive the error bounds for the output in terms of the approximation errors in the input matrix. In Section 3, structure-preserving model reduction methods for second-order LTI systems are briefly discussed. In Section 4, we report some results of numerical experiments for the Euler-Bernoulli beam model.

Throughout this paper, the Euclidean vector norm is denoted by $\|\cdot\|$. Furthermore, we use $\|\cdot\|_{2}$ and $\|\cdot\|_{F}$ to denote the spectral and Frobenius matrix norm, respectively. For the matrix-valued functions $F:[0, T] \rightarrow \mathbb{R}^{n \times m}$ and $H: \mathbb{C} \rightarrow \mathbb{C}^{n \times m}$, we introduce the following norms

$$
\begin{aligned}
& \|F\|_{\mathcal{L}_{2}(0, T)}=\left(\int_{0}^{T}\|F(t)\|_{2}^{2} d t\right)^{1 / 2}, \quad\|F\|_{\mathcal{L}_{\infty}(0, T)}=\sup _{t \in[0, T]}\|F(t)\|_{2}, \\
& \|H\|_{\mathcal{H}_{2}}=\left(\int_{-\infty}^{\infty}\|H(\imath \omega)\|_{F}^{2} d \omega\right)^{1 / 2}, \quad\|H\|_{\mathcal{H}_{\infty}}=\sup _{\omega \in \mathbb{R}}\|H(\imath \omega)\|_{2},
\end{aligned}
$$

where $\imath=\sqrt{-1}$ is the imaginary unit.

2. Approximation of the input matrix. Consider the second-order LTV system (1.1), where the entries of the input matrix $B(t)$ are given in (1.3). Our goal is to 
approximate the columns of $B(t)=\left[b_{1}(t), \ldots, b_{m}(t)\right]$ in a lower dimensional subspace as

$$
b_{j}(t) \approx \sum_{i=1}^{n} \widehat{b}_{i} \psi_{i}\left(\xi_{j}(t)\right)=\widehat{B} \psi\left(\xi_{j}(t)\right), \quad j=1, \ldots, m,
$$

where $\widehat{B}=\left[\widehat{b}_{1}, \ldots, \widehat{b}_{n}\right] \in \mathbb{R}^{N \times n}$ and $\psi\left(\xi_{j}(t)\right)=\left[\psi_{1}\left(\xi_{j}(t)\right), \ldots, \psi_{n}\left(\xi_{j}(t)\right)\right]^{T}$ with $n \ll N$. Then system (1.1) is approximated by a system

$$
\begin{aligned}
M \ddot{\widetilde{q}}(t)+D \dot{\widehat{q}}(t)+K \widehat{q}(t) & =\widehat{B} \widehat{u}(t), \\
\widehat{y}(t) & =C(t) \widehat{q}(t),
\end{aligned}
$$

with the new input $\widehat{u}(t)=\Psi(t) u(t) \in \mathbb{R}^{n}$, where

$$
\Psi(t)=\left[\psi\left(\xi_{1}(t)\right), \ldots, \psi\left(\xi_{m}(t)\right)\right] .
$$

Thus, the state $\widehat{q}(t)$ of system (2.2) approximates the state $q(t)$ of system (1.1), and, as a consequence, the output $\widehat{y}(t)$ approximates the output $y(t)$. Note that the matrix $\widehat{B}$ and the vector-valued function $\psi$ are chosen the same for the approximation of all columns of $B(t)$. Therefore, the approximation problem (2.1) can be rewritten as

$$
B(t) \approx \widehat{B} \Psi(t) .
$$

We now derive the $\mathcal{L}_{\infty}$-norm and $\mathcal{L}_{2}$-norm error bounds for the approximate output $\widehat{y}(t)$ in terms of the error $\|B-\widehat{B} \Psi\|_{\mathcal{L}_{2}(0, T)}$.

THEOREM 2.1. Consider system (1.1) with an initial conditions $q(0)=q_{0}$, $\dot{q}(0)=q_{1}$ and an approximate system (2.2) with the same initial conditions $\widehat{q}(0)=q_{0}$, $\dot{\widehat{q}}(0)=q_{1}$. Assume that all eigenvalues of the pencil $\lambda^{2} M+\lambda D+K$ have negative real part. Then the $\mathcal{L}_{\infty}$-norm and $\mathcal{L}_{2}$-norm of the error in the output can be estimated as

$$
\begin{aligned}
\|y-\widehat{y}\|_{\mathcal{L}_{\infty}(0, T)} & \leq \alpha\|B-\widehat{B} \Psi\|_{\mathcal{L}_{2}(0, T)}\|u\|_{\mathcal{L}_{\infty}(0, T)} \\
\|y-\widehat{y}\|_{\mathcal{L}_{2}(0, T)} & \leq \alpha \sqrt{T}\|B-\widehat{B} \Psi\|_{\mathcal{L}_{2}(0, T)}\|u\|_{\mathcal{L}_{\infty}(0, T)},
\end{aligned}
$$

with a constant $\alpha>0$.

Proof. First, we estimate the error in the approximate state $\widehat{q}(t)$. For this purpose, we transform the second-order system (1.1) into a first-order form

$$
\mathcal{E} \dot{z}(t)=\mathcal{A} z(t)+\mathcal{B}(t) u(t), \quad z(0)=z_{0},
$$

where $z(t)=\left[q^{T}(t), \dot{q}^{T}(t)\right]^{T}, z_{0}=\left[q_{0}^{T}, q_{1}^{T}\right]^{T}$ and

$$
\mathcal{E}=\left[\begin{array}{cc}
D & M \\
M & 0
\end{array}\right], \quad \mathcal{A}=\left[\begin{array}{cc}
-K & 0 \\
0 & M
\end{array}\right], \quad \mathcal{B}(t)=\left[\begin{array}{c}
B(t) \\
0
\end{array}\right] .
$$

Then $\mathcal{B}(t) u(t)$ is approximated by $\mathcal{B}(t) u(t) \approx \widehat{\mathcal{B}} \widehat{u}(t)$ with the matrix $\widehat{\mathcal{B}}=\left[\widehat{B}^{T}, 0\right]^{T}$. Let $\widehat{z}(t)=\left[\widehat{q}^{T}(t), \dot{\widehat{q}}^{T}(t)\right]^{T}$ be a solution of the system

$$
\mathcal{E} \dot{\bar{z}}(t)=\mathcal{A} \widehat{z}(t)+\widehat{\mathcal{B}} \widehat{u}(t), \quad \widehat{z}(0)=z_{0} .
$$

Since systems (2.7) and (2.8) have the same initial conditions, an error vector

$$
\epsilon_{z}(t)=z(t)-\widehat{z}(t)
$$


has the form

$$
\epsilon_{z}(t)=\int_{0}^{t} e^{\mathcal{E}^{-1} \mathcal{A}(t-\tau)} \mathcal{E}^{-1}(\mathcal{B}(\tau) u(\tau)-\widehat{\mathcal{B}} \widehat{u}(\tau)) d \tau .
$$

Then the norm of $\epsilon_{z}(t)$ can be estimated as

$$
\begin{aligned}
\left\|\epsilon_{z}(t)\right\|^{2} & \leq\left(\int_{0}^{t}\left\|e^{\mathcal{E}^{-1} \mathcal{A}(t-\tau)}\right\|_{2}\left\|\mathcal{E}^{-1}\right\|_{2}\|\mathcal{B}(\tau) u(\tau)-\widehat{\mathcal{B}} \widehat{u}(\tau)\| d \tau\right)^{2} \\
& \leq\left\|\mathcal{E}^{-1}\right\|_{2}^{2} \int_{0}^{t}\left\|e^{\mathcal{E}^{-1} \mathcal{A}(t-\tau)}\right\|_{2}^{2} d \tau \int_{0}^{t}\|B(\tau) u(\tau)-\widehat{B} \widehat{u}(\tau)\|^{2} d \tau .
\end{aligned}
$$

Since all eigenvalues of $\lambda^{2} M+\lambda D+K$ have negative real part, all eigenvalues of the pencil $\lambda \mathcal{E}-\mathcal{A}$ have also negative real part, and, hence, there exist constants $\vartheta, \kappa>0$ such that

$$
\left\|e^{\mathcal{E}^{-1} \mathcal{A} t}\right\|_{2} \leq \vartheta e^{-\kappa t}, \quad t \geq 0,
$$

see $[11,27,38]$. Then we have

$$
\int_{0}^{t}\left\|e^{\mathcal{E}^{-1} \mathcal{A}(t-\tau)}\right\|_{2}^{2} d \tau \leq \vartheta^{2} \int_{0}^{t} e^{-2 \kappa(t-\tau)} d \tau=\frac{\vartheta^{2}}{2 \kappa}\left(1-e^{-2 \kappa t}\right) .
$$

Introducing an error vector $\epsilon_{q}(t)=q(t)-\widehat{q}(t)$ and using the estimate $\left\|\epsilon_{q}(t)\right\| \leq\left\|\epsilon_{z}(t)\right\|$, we have

$$
\begin{aligned}
\|y(t)-\widehat{y}(t)\| & =\left\|C(t) \epsilon_{q}(t)\right\| \leq\|C(t)\|_{2}\left\|\epsilon_{z}(t)\right\| \\
& \leq \frac{\vartheta}{\sqrt{2 \kappa}}\left\|\mathcal{E}^{-1}\right\|_{2}\|C(t)\|_{2} \sqrt{1-e^{-2 \kappa t}}\|B u-\widehat{B} \widehat{u}\|_{\mathcal{L}_{2}(0, T)}
\end{aligned}
$$

for all $t \geq 0$. Then

$$
\|y-\widehat{y}\|_{\mathcal{L}_{\infty}(0, T)} \leq \frac{\vartheta}{\sqrt{2 \kappa}}\left\|\mathcal{E}^{-1}\right\|_{2}\|C\|_{\mathcal{L}_{\infty}(0, T)}\|B u-\widehat{B} \widehat{u}\|_{\mathcal{L}_{2}(0, T)}
$$

and

$$
\|y-\widehat{y}\|_{\mathcal{L}_{2}(0, T)} \leq \frac{\vartheta \sqrt{T}}{\sqrt{2 \kappa}}\left\|\mathcal{E}^{-1}\right\|_{2}\|C\|_{\mathcal{L}_{\infty}(0, T)}\|B u-\widehat{B} \widehat{u}\|_{\mathcal{L}_{2}(0, T)} .
$$

Finally, taking into account that

$$
\|B u-\widehat{B} \widehat{u}\|_{\mathcal{L}_{2}(0, T)} \leq\|B-\widehat{B} \Psi\|_{\mathcal{L}_{2}(0, T)}\|u\|_{\mathcal{L}_{\infty}(0, T)},
$$

we obtain the estimates (2.5) and (2.6) with the constant

$$
\alpha=\frac{\vartheta}{\sqrt{2 \kappa}}\left\|\mathcal{E}^{-1}\right\|_{2}\|C\|_{\mathcal{L}_{\infty}(0, T)} .
$$

Estimates (2.5) and (2.6) show that the small approximation error $\|B-\widehat{B} \Psi\|_{\mathcal{L}_{2}(0, T)}$ implies the small error in the output. This suggests to reformulate the approximation problem (2.4) as a linear least-squares (LLS) problem in two different ways: 
(P1) Given a matrix $\widehat{B}$, find a vector-valued function $\psi$ such that the error $\|B-\widehat{B} \Psi\|_{\mathcal{L}_{2}(0, T)}$ with $\Psi$ as in $(2.3)$ is as small as possible;

(P2) Given a vector-valued function $\psi$, find a matrix $\widehat{B}$ such that the error $\|B-\widehat{B} \Psi\|_{\mathcal{L}_{2}(0, T)}$ with $\Psi$ as in (2.3) is as small as possible.

A possible approach for solving the approximation problem (P1) is an empirical interpolation method (EIM) from [4] adapted for our purpose. In this approach, the construction of the basis matrix $\widehat{B}$ and a set of interpolation points $\left\{t_{1}, \ldots, t_{n}\right\}$ is based on a greedy algorithm combined with a least-squares approximation. Let $\mathcal{T} \subset[0, T]$ be a finite but sufficiently large set of time samples. First, we choose

$$
t_{1}=\arg \max _{t \in \mathcal{T}}\|B(t)\|_{F}
$$

and define $\widehat{B}_{1}=B\left(t_{1}\right)$. Then for $j=1, \ldots, n-1$, we compute the least-squares approximation $B(t) \approx \widehat{B}_{j} \Psi_{j}(t)$ with $\Psi_{j}(t)=\left(\widehat{B}_{j}^{T} \widehat{B}_{j}\right)^{-1} \widehat{B}_{j}^{T} B(t)$, determine the next interpolation point

$$
t_{j+1}=\arg \max _{t \in \mathcal{T}}\left\|B(t)-\widehat{B}_{j} \Psi_{j}(t)\right\|_{F}
$$

and update the basis matrix $\widehat{B}_{j+1}=\left[\widehat{B}_{j}, B\left(t_{j+1}\right)\right]$. Finally, we get the approximation $B(t) \approx \widehat{B} \Psi(t)$ with $\widehat{B}=\widehat{B}_{n}$ and $\Psi(t)=\left(\widehat{B}^{T} \widehat{B}\right)^{-1} \widehat{B}^{T} B(t)$ which satisfies the interpolation conditions $B\left(t_{j}\right)=\widehat{B} \Psi\left(t_{j}\right)$ for $j=1, \ldots, n$. The numerical experiments for the Euler-Bernoulli model show, however, that this approach provides a satisfactory approximation only if the number $n$ of columns of $\widehat{B}$ is sufficiently large. For small $n$, the approximation error in the output is unacceptably large, see Section 4.

In what follows, we consider the minimization problem (P2) only remaining the first one for future work.

REMARK 1. We can also approximate the rows of the output matrix

$$
C(t)=\left[c_{1}^{T}(t), \ldots, c_{p}^{T}(t)\right]^{T}
$$

as

$$
c_{j}(t) \approx \sum_{i=1}^{l} \widehat{c}_{i} \zeta_{i}\left(\eta_{j}(t)\right)=\zeta\left(\eta_{j}(t)\right) \widehat{C}, \quad j=1, \ldots, p,
$$

where $\widehat{C}=\left[\widehat{c}_{1}^{T}, \ldots, \widehat{c}_{l}^{T}\right]^{T} \in \mathbb{R}^{l \times N}, \zeta\left(\eta_{j}(t)\right)=\left[\zeta_{1}\left(\eta_{j}(t)\right), \ldots, \zeta_{l}\left(\eta_{j}(t)\right)\right]$ with $l \ll N$, and $\eta_{j}(t)$ is a position of the $j$-th observation. In this case, the output of system (1.1) is approximated by $y(t) \approx\left[\zeta^{T}\left(\eta_{1}(t)\right), \ldots, \zeta^{T}\left(\eta_{p}(t)\right)\right]^{T} \widehat{\widehat{y}}(t)$, where $\widehat{\widehat{y}}(t)$ is the output of the LTI system

$$
\begin{aligned}
M \ddot{\widehat{q}}(t)+D \dot{\hat{q}}(t)+K \underset{\widehat{\widehat{q}}}{\widehat{\widehat{y}}(t)}(t)=\widehat{\widehat{B}} \widehat{u}(t) \\
\widehat{C} \widehat{q}(t)
\end{aligned}
$$

However, in practice, we do not approximate the output matrix to avoid an additional error in the output.

2.1. Solving the linear least-squares problem. For simplicity, we restrict ourselves to the singe-input case $m=1$ with the input matrix

$$
B(t)=\left[\begin{array}{c}
\varphi_{N, 1}(\xi(t)) \\
\vdots \\
\varphi_{N, N}(\xi(t))
\end{array}\right],
$$


where $\varphi_{N, j}(x)$ are the FEM basis functions and the trajectory $\xi(t)$ of the moving load is assumed to be known for all $t \in[0, T]$. An extension to the multiple-input case is straightforward. In order to solve the minimization problem (P2), we define the cost functional

$$
\mathcal{J}(\widehat{B})=\|B-\widehat{B} \Psi\|_{\mathcal{L}_{2}(0, T)}^{2}=\int_{0}^{T}\|B(t)-\widehat{B} \psi(\xi(t))\|^{2} d t
$$

with $\Psi(t)=\psi(\xi(t))=\left[\psi_{1}(\xi(t)), \ldots, \psi_{n}(\xi(t))\right]^{T}$. We aim to find $\widehat{B} \in \mathbb{R}^{N \times n}$ such that $\mathcal{J}(\widehat{B})$ is minimized. Differentiating $\mathcal{J}$ with respect to the entries of $\widehat{B}=\left[\widehat{b}_{i k}\right]$, we get

$$
\frac{\partial}{\partial \widehat{b}_{i k}} \mathcal{J}(\widehat{B})=-2 \int_{0}^{T}\left(\varphi_{N, i}(\xi(t)) \psi_{k}(\xi(t))-\left(\sum_{j=1}^{n} \widehat{b}_{i j} \psi_{j}(\xi(t))\right) \psi_{k}(\xi(t))\right) d t
$$

for $i=1, \ldots, N$ and $k=1, \ldots, n$. Requiring for each partial derivative to be zero, we obtain the normal equation $\widehat{B} F=G$, where the matrices $G=\left[g_{i k}\right] \in \mathbb{R}^{N \times n}$ and $F=\left[f_{j k}\right] \in \mathbb{R}^{n \times n}$ have the entries

$$
g_{i k}=\int_{0}^{T} \varphi_{N, i}(\xi(t)) \psi_{k}(\xi(t)) d t, \quad f_{j k}=\int_{0}^{T} \psi_{j}(\xi(t)) \psi_{k}(\xi(t)) d t,
$$

respectively, for $i=1, \ldots, N$ and $j, k=1, \ldots, n$. If $F$ is positive definite, then the solution of the LLS problem (P2) is given by $\widehat{B}=G F^{-1}$. Next we consider two possible choices for the function $\psi$.

2.1.1. Approximation with orthogonal polynomials. A particular choice of the LLS approximation is a truncated expansion in a system of orthogonal polynomials, also known as Fourier series in orthogonal polynomials $[28,30]$. Let $p_{j}(\theta)$, $j=0,1,2, \ldots$, form a system of orthogonal polynomials on an interval $[a, b]$ with a weight function $\rho(\theta)>0$. Each polynomial $p_{j}(\theta)$ is of degree $j$ and satisfies the orthogonality conditions

$$
\int_{a}^{b} \rho(\theta) p_{k}(\theta) p_{j}(\theta) d \theta=\gamma_{k} \delta_{k j}
$$

where $\gamma_{k}>0$ and $\delta_{k j}$ is the Kronecker delta. Then $\varphi_{N, i}(\xi(t))$ as the function of $t$ can be expanded as

$$
\varphi_{N, i}(\xi(t))=\sum_{k=0}^{\infty} c_{i k} p_{k}(\theta(t))
$$

where $\theta:[0, T] \rightarrow[a, b]$ and the coefficients $c_{i k}$ have the form

$$
c_{i k}=\frac{1}{\gamma_{k}} \int_{0}^{T} \rho(\theta(t)) \varphi_{N, i}(\xi(t)) p_{k}(\theta(t)) \dot{\theta}(t) d t .
$$

One can show [30, Section 10.1] that a partial sum

$$
\sum_{k=0}^{n-1} c_{i k} p_{k}(\theta(t))
$$


gives a best approximation to $\varphi_{N, i}(\xi(t))$ that minimizes

$$
\int_{0}^{T} \rho(\theta(t))\left(\varphi_{N, i}(\xi(t))-\sum_{k=0}^{n-1} c_{i k} p_{k}(\theta(t))\right)^{2} \dot{\theta}(t) d t
$$

In particular, one can use the Legendre polynomials $p_{k}(\theta)$, which are orthogonal on the interval $[a, b]=[-1,1]$ with the weight function $\rho(\theta) \equiv 1$. They satisfy the orthogonality conditions

$$
\int_{-1}^{1} p_{k}(\theta) p_{j}(\theta) d \theta=\frac{2}{2 k+1} \delta_{k j}
$$

Then the entries of the matrix $\widehat{B}$ are determined as

$$
\widehat{b}_{i j}=\frac{2(j-1)+1}{T} \int_{0}^{T} \varphi_{N, i}(\xi(t)) p_{j-1}(\theta(t)) d t
$$

with $\theta(t)=(2 t-T) / T$. Thus, $\widehat{B} \Psi(t)$ with $\Psi(t)=\left[p_{0}(\theta(t)), \ldots, p_{n-1}(\theta(t))\right]^{T}$ provides the LLS approximation to $B(t)=\varphi(\xi(t))$. The fact that the weight function in the case of the Legendre polynomials is equal to unity makes all times $t$ in $[0, T]$ equivalent in the approximation, i.e., they give the same contribution to the approximation. This is not necessarily fulfilled, for example, for the Chebyshev polynomials, for which the weight function is $\rho(\theta)=1 / \sqrt{1-\theta^{2}}$.

2.1.2. Approximation by the FEM coarse grid basis functions. Another choice for the function $\psi$ in the LLS problem (P2) is offered by the FEM basis functions on a coarse grid. Let the coarse grid have $n$ degrees of freedom and let $\varphi_{n, i}(x)$ denote the corresponding FEM basis functions. For $\psi(\xi(t))=\left[\varphi_{n, 1}(\xi(t)), \ldots, \varphi_{n, n}(\xi(t))\right]^{T}$, we determine $\widehat{B}=G F^{-1}$ with $G$ and $F$ as in (2.12). One can expect that the closer $n$ to $N$, the smaller the error $\|B-\widehat{B} \Psi\|_{\mathcal{L}_{2}(0, T)}$ is.

2.2. Interpolation by B-splines. An alternative way to solve the approximation problem (2.1) is interpolation. It can be carried out, for example, by cubic B-splines $[12,21]$. Let the time interval $[0, T] \subset \mathbb{R}$ be partitioned into $n-3$ subintervals with the knots $0=t_{0}<t_{1}<\ldots<t_{n-3}=T$ and let

$$
t_{-3}<t_{-2}<t_{-1}<0, \quad T<t_{n-2}<t_{n-1}<t_{n}
$$

be additional knots. The cubic B-splines are the twice continuously differentiable functions of the form

$$
\beta_{j}(t)= \begin{cases}\beta_{j, 1}(t), & \text { if } t \in\left[t_{j-2}, t_{j-1}\right] \\ \beta_{j, 2}(t), & \text { if } t \in\left[t_{j-1}, t_{j}\right] \\ \beta_{j, 3}(t), & \text { if } t \in\left[t_{j}, t_{j+1}\right] \\ \beta_{j, 4}(t), & \text { if } t \in\left[t_{j+1}, t_{j+2}\right] \\ 0 & \text { otherwise }\end{cases}
$$


where $\beta_{j, k}(t), k=1,2,3,4$, are polynomials of degree 3 . For an equidistant grid with a stepsize $\tau=T /(n-3)$ these polynomials are given by

$$
\begin{aligned}
& \beta_{j, 1}(t)=\frac{1}{6 \tau^{3}}\left(t-t_{j-2}\right)^{3}, \\
& \beta_{j, 2}(t)=\frac{1}{6 \tau^{3}}\left(3\left(t_{j-1}-t\right)^{3}+3 \tau\left(t_{j-1}-t\right)^{2}-3 \tau^{2}\left(t_{j-1}-t\right)+\tau^{3}\right), \\
& \beta_{j, 3}(t)=\frac{1}{6 \tau^{3}}\left(3\left(t-t_{j+1}\right)^{3}+3 \tau\left(t-t_{j+1}\right)^{2}-3 \tau^{2}\left(t-t_{j+1}\right)+\tau^{3}\right), \\
& \beta_{j, 4}(t)=\frac{1}{6 \tau^{3}}\left(t_{j+2}-t\right)^{3} .
\end{aligned}
$$

We now construct a cubic interpolant for the function $B(t)$ in $(2.11)$. We are seeking for an approximation with a cubic spline

$$
B(t) \approx S(t)=\sum_{j=1}^{n} \widehat{b}_{j} \beta_{j-2}(t)
$$

satisfying the interpolation conditions

$$
S\left(t_{i}\right)=B\left(t_{i}\right)=\varphi\left(\xi\left(t_{i}\right)\right), \quad i=0, \ldots, n-3 .
$$

In order to ensure the uniqueness of the interpolant, we impose, for example, the "nota-knot" end conditions by requiring that the third derivative of $S(t)$ is continuous at $t_{1}$ and $t_{n-4}$. These conditions together with (2.13) yield a linear system

$$
\left[\widehat{b}_{1}, \ldots, \widehat{b}_{n}\right]\left[s_{1}, \mathcal{S}, s_{n}\right]=\left[0, \varphi\left(\xi\left(t_{0}\right)\right), \ldots, \varphi\left(\xi\left(t_{n-3}\right)\right), 0\right]
$$

where

$$
\begin{aligned}
\mathcal{S} & =\left[\begin{array}{cccc}
\beta_{-1}\left(t_{0}\right) & & \\
\beta_{0}\left(t_{0}\right) & \beta_{0}\left(t_{1}\right) & & \\
\beta_{1}\left(t_{0}\right) & \beta_{1}\left(t_{1}\right) & \ddots & \beta_{n-4}\left(t_{n-3}\right) \\
& \beta_{2}\left(t_{1}\right) & \ddots & \beta_{n-3}\left(t_{n-3}\right) \\
& \ddots & \beta_{n-2}\left(t_{n-3}\right)
\end{array}\right] \in \mathbb{R}^{n \times(n-2)}, \\
& \\
s_{1} & =\left[\begin{array}{ll}
s_{11}, s_{21}, s_{31}, s_{41}, s_{51}, 0, \ldots, 0 \\
s_{n}
\end{array}\right. \\
s_{n} & =\left[0, \ldots, 0, s_{n-4, n}, s_{n-3, n}, s_{n-2, n}, s_{n-1, n}, s_{n n}\right]^{T}
\end{aligned}
$$

with

$$
\begin{aligned}
s_{11} & =\beta_{-1,4}^{\prime \prime \prime}\left(t_{1}\right), & s_{n-4, n} & =\beta_{n-6,4}^{\prime \prime \prime}\left(t_{n-4}\right), \\
s_{21} & =\beta_{0,3}^{\prime \prime \prime}\left(t_{1}\right)-\beta_{0,4}^{\prime \prime \prime}\left(t_{1}\right), & s_{n-3, n} & =\beta_{n-5,3}^{\prime \prime \prime}\left(t_{n-4}\right)-\beta_{n-5,4}^{\prime \prime \prime}\left(t_{n-4}\right), \\
s_{31} & =\beta_{1,2}^{\prime \prime \prime}\left(t_{1}\right)-\beta_{1,3}^{\prime \prime \prime}\left(t_{1}\right), & s_{n-2, n} & =\beta_{n-4,2}^{\prime \prime \prime}\left(t_{n-4}\right)-\beta_{n-4,3}^{\prime \prime \prime}\left(t_{n-4}\right), \\
s_{41} & =\beta_{2,1}^{\prime \prime \prime}\left(t_{1}\right)-\beta_{2,2}^{\prime \prime \prime}\left(t_{1}\right), & s_{n-1, n} & =\beta_{n-3,1}^{\prime \prime \prime}\left(t_{n-4}\right)-\beta_{n-3,2}^{\prime \prime \prime}\left(t_{n-4}\right), \\
s_{51} & =-\beta_{3,1}^{\prime \prime \prime}\left(t_{1}\right), & s_{n, n} & =-\beta_{n-2,1}^{\prime \prime \prime}\left(t_{n-4}\right) .
\end{aligned}
$$


For the equidistant grid, we get

$$
\begin{aligned}
& \mathcal{S}=\frac{1}{6}\left[\begin{array}{ccccc}
1 & 0 & 0 & \cdots & 0 \\
4 & 1 & 0 & \cdots & 0 \\
1 & 4 & 1 & \cdots & 0 \\
0 & 1 & 4 & \cdots & 0 \\
\vdots & \vdots & \vdots & \ddots & \vdots \\
0 & 0 & 0 & \cdots & 4 \\
0 & 0 & 0 & \cdots & 1
\end{array}\right], \\
& s_{1}=\frac{1}{\tau^{3}}[-1,4,-6,4,-1,0, \ldots, 0]^{T}, \\
& s_{n}=\frac{1}{\tau^{3}}[0, \ldots, 0,-1,4,-6,4,-1]^{T} .
\end{aligned}
$$

If $\left[s_{1}, \mathcal{S}, s_{n}\right]$ is nonsingular, then the matrix $\widehat{B}$ can be determined as

$$
\widehat{B}=\left[\widehat{b}_{1}, \ldots, \widehat{b}_{n}\right]=\left[0, \varphi\left(\xi\left(t_{0}\right)\right), \ldots, \varphi\left(\xi\left(t_{n-3}\right)\right), 0\right]\left[s_{1}, \mathcal{S}, s_{n}\right]^{-1} .
$$

Note that B-splines can also be used to solve the LLS problem (P2) as described in Section 2.1.

3. Model reduction of mechanical systems. In this section, we briefly review structure-preserving model reduction of second-order systems. Approximating the input matrix as in (2.4) we get the second-order LTI system

$$
\begin{aligned}
M \ddot{\widetilde{q}}(t)+D \dot{\widehat{q}}(t)+K \widehat{q}(t) & =\widehat{B} \widehat{u}(t), \\
\widehat{y}_{I}(t) & =\widehat{q}(t),
\end{aligned}
$$

with $\widehat{u}(t)=\Psi(t) u(t)$. Replacing this system by a reduced model

$$
\begin{aligned}
\widetilde{M} \ddot{\widetilde{q}}(t)+\widetilde{D} \dot{\widetilde{q}}(t)+\widetilde{K} \widetilde{q}(t) & =\widetilde{B} \widehat{u}(t), \\
\widetilde{y}_{I}(t) & =\widetilde{C} \widetilde{q}(t),
\end{aligned}
$$

with the reduced matrices

$$
\widetilde{M}=V^{T} M V, \quad \widetilde{D}=V^{T} D V, \quad \widetilde{K}=V^{T} K V, \quad \widetilde{B}=V^{T} \widehat{B}, \quad \widetilde{C}=V,
$$

and $V \in \mathbb{R}^{N \times r}$, we approximate the input $y(t)$ of system (1.1) by

$$
\widetilde{y}(t)=C(t) \widetilde{y}_{I}(t)=C(t) V \widetilde{q}(t) .
$$

Note that the use of one-sided projection guarantees the preservation of symmetry and positive definiteness in $\widetilde{M}, \widetilde{D}$ and $\widetilde{K}$ for symmetric positive definite $M, D$ and $K$.

Let

$$
H(s)=\left(s^{2} M+s D+K\right)^{-1} \widehat{B}, \quad \widetilde{H}(s)=\widetilde{C}\left(s^{2} \widetilde{M}+s \widetilde{D}+\widetilde{K}\right)^{-1} \widetilde{B}
$$

be the transfer functions of systems (3.1) and (3.2), respectively. Then the error in the output can be estimated by

$$
\begin{aligned}
\left\|\widehat{y}_{I}(t)-\widetilde{y}_{I}(t)\right\|_{\mathcal{L}_{\infty}(0, \infty)} & \leq\|H-\widetilde{H}\|_{\mathcal{H}_{2}}\|\widehat{u}\|_{\mathcal{L}_{2}(0, \infty)}, \\
\left\|\widehat{y}_{I}(t)-\widetilde{y}_{I}(t)\right\|_{\mathcal{L}_{2}(0, \infty)} & \leq\|H-\widetilde{H}\|_{\mathcal{H}_{\infty}}\|\widehat{u}\|_{\mathcal{L}_{2}(0, \infty)}
\end{aligned}
$$


where $\mathcal{H}_{2}$-norm and $\mathcal{H}_{\infty}$-norm are defined in (1.6), see [1]. Combining the approximation of the input matrix as described in Section 2 with model reduction of the LTI system (3.1), we obtain the following estimates for the total error in the output.

THEOREM 3.1. Consider the second-order system (1.1) and the reduced system (3.2a) with the output $\widetilde{y}(t)=C(t) V \widetilde{q}(t)$. The the $\mathcal{L}_{\infty}$-norm and $\mathcal{L}_{2}$-norm of the error $y-\widetilde{y}$ can be estimated as

$$
\begin{aligned}
\|y-\widetilde{y}\|_{\mathcal{L}_{\infty}(0, T)} \leq \alpha & \|B-\widehat{B} \Psi\|_{\mathcal{L}_{2}(0, T)}\|u\|_{\mathcal{L}_{\infty}(0, T)} \\
& +\|C\|_{\mathcal{L}_{\infty}(0, T)}\|H-\widetilde{H}\|_{\mathcal{H}_{2}}\|\widehat{u}\|_{\mathcal{L}_{2}(0, \infty)}, \\
\|y-\widetilde{y}\|_{\mathcal{L}_{2}(0, T) \leq \alpha} & \sqrt{T}\|B-\widehat{B} \Psi\|_{\mathcal{L}_{2}(0, T)}\|u\|_{\mathcal{L}_{\infty}(0, T)} \\
& +\|C\|_{\mathcal{L}_{\infty}(0, T)}\|H-\widetilde{H}\|_{\mathcal{H}_{\infty}}\|\widehat{u}\|_{\mathcal{L}_{2}(0, \infty)},
\end{aligned}
$$

where $\alpha$ is as in (2.10).

Proof. For $\widehat{y}(t)=C(t) \widehat{y}_{I}(t)$, we have

$$
\|y-\widetilde{y}\|_{\mathcal{L}_{\infty}(0, T)} \leq\|y-\widehat{y}\|_{\mathcal{L}_{\infty}(0, T)}+\left\|C \widehat{y}_{I}-C \widetilde{y}_{I}\right\|_{\mathcal{L}_{\infty}(0, T)} .
$$

The the estimate (3.6) immediately follows from (2.5) and (3.4). The estimate (3.7) can be proved analogously using (2.6) and (3.5).

For model reduction of LTI systems, many different model reduction methods have been developed in the last four decades, see $[1,35]$. One of the most popular approaches is balanced truncation. It is based on balancing the controllability and observability Gramians which solve dual Lyapunov equations [26]. Transforming the second-order system (3.1) into the first-order form and applying balanced truncation, one gets the reduced system with a computable error bound on the $\mathcal{H}_{\infty}$-norm of the transfer function. Structure-preserving balanced truncation for second-order systems has been considered in $[25,31]$. This approach relies on balancing the position and velocity controllability and observability Gramians defined by the diagonal blocks of the Gramians of the corresponding first-order system of doubled dimension. For solving large-scale Lyapunov equations, a second-order low-rank alternating directions implicit (SO-LR-ADI) method has been developed in $[8,9]$ which employs the secondorder structure of the matrix coefficients. It should, however, be noticed that, unlike the first-order version, second-order balanced truncation is lacking the error bound. Another difficulty is that the SO-LR-ADI method has a rather slow convergence for mechanical systems with a weak damping.

Alternative model reduction approaches are moment matching approximation and tangential interpolation which are based on (rational) Krylov subspace methods, e.g., $[2,18]$. Structure-preserving Krylov-based algorithms for second-order systems have been presented in $[3,6,16,33]$. The approximation quality of these methods strongly depends on the choice of interpolation points. Iterative Krylov subspace methods with an adaptive choice of interpolation points have been developed in [23, 19]. An extension of these methods to second-order systems can be found in [37, 40].

For model reduction of system (3.1), we use a modification of the second-order iterative rational Krylov algorithm (SO-IRKA) presented in [40]. In this algorithm, the projection matrix is constructed iteratively in the form

$$
V_{j}=\left[\left(\sigma_{1 j}^{2} M+\sigma_{1 j} D+K\right)^{-1} \widehat{B} w_{1 j}, \ldots,\left(\sigma_{r j}^{2} M+\sigma_{r j} D+K\right)^{-1} \widehat{B} w_{r j}\right],
$$

where the interpolation points $\sigma_{1 j}, \ldots, \sigma_{r j} \in \mathbb{C}$ are selected from the mirror images of $2 r$ eigenvalues of the reduced pencil

$$
\lambda^{2} V_{j-1}^{T} M V_{j-1}+\lambda V_{j-1}^{T} D V_{j-1}+V_{j-1}^{T} K V_{j-1}
$$


closest to the imaginary axis, and the tangential directions $w_{1 j}, \ldots, w_{r j}$ are determined from the corresponding eigenvectors, see [40, Section 5.3.2] for details. Our modification, called M-SO-IRKA, is based on the observation in [18] that the use of imaginary interpolation points seems to be the most natural if one is interested in minimizing the frequency response error $H(\iota \omega)-\widetilde{H}(\iota \omega)$. Therefore, we choose the interpolation points $\sigma_{k j}$ as the positive imaginary parts of the eigenvalues of the reduced pencil in (3.8). Such a choice is justified by the numerical experiments presented in the next section. In order to keep the reduced model real, we determine the projection matrix $V$ with orthogonal columns from a rank-revealing QR decomposition of the matrix $\left[\operatorname{Re}\left(V_{j}\right), \operatorname{Im}\left(V_{j}\right)\right]$.

4. Numerical example. We consider the vibration of a simply supported EulerBernoulli beam of length $l$ subjected to a point force moving along the beam with a constant velocity $v$. The transverse displacement of the beam is described by a function $w(x, t)$ satisfying Euler-Bernoulli equation (1.2) with $f(x, t)=-\delta(x-\xi(t)) u$, where $u>0$ is the constant magnitude and $\xi(t)=v t$ is the instantaneous position of the acting force at time $t \in[0, T], T=l / v$, see [17]. The model parameters are collected in Table 1.

TABLE 4.1

Beam and load parameters

\begin{tabular}{|c|c|}
\hline Parameter & Value \\
\hline$l$ & $50 \mathrm{~m}$ \\
$\mu A$ & $4650 \mathrm{~kg} / \mathrm{m}$ \\
$\omega_{\mathrm{d}}$ & $1000 \mathrm{~m}^{3} \mathrm{~Hz} /\left(4 l^{2}\left(0.3 \mathrm{~m}-1.2 \times 10^{-3} l\right)\right)$ \\
$E J$ & $\mu A(500 l / \pi)^{2} \mathrm{~Hz}^{2} \mathrm{~m}^{2}$ \\
$u$ & $1.96133 \times 10^{5} \mathrm{~N}$ \\
$v$ & $40 \mathrm{~km} / \mathrm{h}(\approx 11 \mathrm{~m} / \mathrm{s})$ \\
\hline
\end{tabular}

For the FEM discretization of the Euler-Bernoulli equation (1.2), we partition the interval $[0, l]$ into $n_{x}=l / h$ subintervals $\left[x_{i}, x_{i+1}\right]$ of length $h$ with $x_{i}=i h$ for $i=0, \ldots, n_{x}$. Then $w(x, t)$ can be approximated by

$$
w_{h}(x, t)=\theta_{0}(t) \phi_{0}^{(1)}(x)+\sum_{i=1}^{n_{x}-1}\left(w_{i}(t) \phi_{i}^{(0)}(x)+\theta_{i}(t) \phi_{i}^{(1)}(x)\right)+\theta_{n_{x}}(t) \phi_{n_{x}}^{(1)}(x)
$$

with the functions

$$
\begin{aligned}
& \phi_{0}^{(1)}(x)= \begin{cases}x-2 x^{2} / h+x^{3} / h^{2} & \text { for } x \in\left[x_{0}, x_{1}\right], \\
0 & \text { otherwise }\end{cases} \\
& \phi_{i}^{(0)}(x)= \begin{cases}1-3(x-i h)^{2} / h^{2}-2(x-i h)^{3} / h^{3} & \text { for } x \in\left[x_{i-1}, x_{i}\right], \\
1-3(x-i h)^{2} / h^{2}+2(x-i h)^{3} / h^{3} & \text { for } x \in\left[x_{i}, x_{i+1}\right], \\
0 & \text { otherwise, }\end{cases} \\
& \phi_{i}^{(1)}(x)= \begin{cases}(x-i h)+2(x-i h)^{2} / h+(x-i h)^{3} / h^{2} & \text { for } x \in\left[x_{i-1}, x_{i}\right], \\
(x-i h)-2(x-i h)^{2} / h+(x-i h)^{3} / h^{2} & \text { for } x \in\left[x_{i}, x_{i+1}\right], \\
0 & \text { otherwise }\end{cases} \\
& \phi_{n_{x}}^{(1)}(x)= \begin{cases}(x-l)+2(x-l)^{2} / h+(x-l)^{3} / h^{2} & \text { for } x \in\left[x_{n_{x}-1}, x_{n_{x}}\right] \\
0 & \text { otherwise }\end{cases}
\end{aligned}
$$


The FEM discretization of (1.2) yields the second-order system (1.1), where

$$
q(t)=\left[\theta_{0}(t), w_{1}(t), \theta_{1}(t), \ldots, w_{n_{x}-1}(t), \theta_{n_{x}-1}(t), \theta_{n_{x}}(t)\right]^{T}
$$

and the entrees of the mass matrix $M=\left[m_{i j}\right]$ and the stiffness matrix $K=\left[k_{i j}\right]$ are defined as

$$
\begin{aligned}
m_{i j} & =\mu A \int_{0}^{l} \varphi_{N, i}(x) \varphi_{N, j}(x) d x \\
k_{i j} & =E J \int_{0}^{l} \frac{d^{2}}{d x^{2}} \varphi_{N, i}(x) \frac{d^{2}}{d x^{2}} \varphi_{N, j}(x) d x
\end{aligned}
$$

for $i, j=1, \ldots, N$, e.g., [7]. Here, $N=2 n_{x}$, and the FEM basis functions have the form

$$
\begin{array}{rlrl}
\varphi_{N, 1}(x) & =\phi_{0}^{(1)}(x), & \varphi_{N, N}(x) & =\phi_{n_{x}}^{(1)}(x), \\
\varphi_{N, 2 i}(x) & =\phi_{i}^{(0)}(x), \quad \varphi_{N, 2 i+1}(x)=\phi_{i}^{(1)}(x), \quad i=1, \ldots, n_{x}-1 .
\end{array}
$$

Furthermore, the damping matrix is proportional to the mass matrix, i.e., $D=2 \omega_{\mathrm{d}} M$. We assume that the observation position coincides with the position of the acting force implying that $C^{T}(t)=B(t)$ with the input matrix $B(t)$ as in (2.11).

In our experiments, the dimension of system (1.1) is $N=5000$. For time integration, we use the trapezoidal rule. Figure 4.1 shows the output $y(t)$. In Figure 4.2, we present the absolute errors $\|y(t)-\widetilde{y}(t)\|$, where $y(t)=C(t) q(t)$ and $\widetilde{y}(t)=C(t) V \widetilde{q}(t)$ are the outputs of (1.1) and the reduced models of different dimensions obtained by applying M-SO-IRKA to system (1.5) with the input matrix being the identity matrix.

In Figures 4.3, 4.9 and 4.13, we present the absolute errors in the output for systems with the approximated input matrix obtained by the LLS method with the FEM basis functions on a coarse grid (LLS-FEM), the LLS method with the Legendre polynomials (LLS-Legendre) and cubic B-spline interpolation (Spline interp.) with $n=30,50$ and 100 . One can observe that all three approximation methods provide very similar results. Moreover, with increasing $n$, we obtain more accurate approximations. In Figure 4.3, we include also the absolute error in the output for the system with the approximated input matrix obtained by the EIM combined with the LLS method (LLS-EIM). This error is much larger than the other errors.

Next, we compare different model reduction techniques for system (2.2) with the input matrix with $n=30$ columns obtained by the LLS-FEM approach. Figure 4.4 shows the spectral norms of the frequency response

$$
H(\iota \omega)=\left((\iota \omega)^{2} M+\iota \omega D+K\right)^{-1} \widehat{B}
$$

and the absolute errors $H(\iota \omega)-\widetilde{H}(\iota \omega)$ for the reduced models obtained by M-SO-IRKA, SO-IRKA and MIRA from [37] with one expansion point. We see that the reduced model computed by MIRA has, in general, a smaller error than the other models, but it has the larger dimension. Comparing M-SO-IRKA and SO-IRKA, we find a significant difference in the error for higher frequencies. In Figure 4.5, we present the absolute errors in the output for three reduced models. One can see that M-SO-IRKA provides the best result with respect to the reduced dimension and the error in comparison with SO-IRKA and MIRA. Therefore, in the following, we use only M-SO-IRKA for model reduction of system (2.2).

Figures 4.6, 4.7 and 4.8 show the absolute errors in the output for the reduced models of dimension $r=15,20,25$ and 30 obtained by the approximation of the 


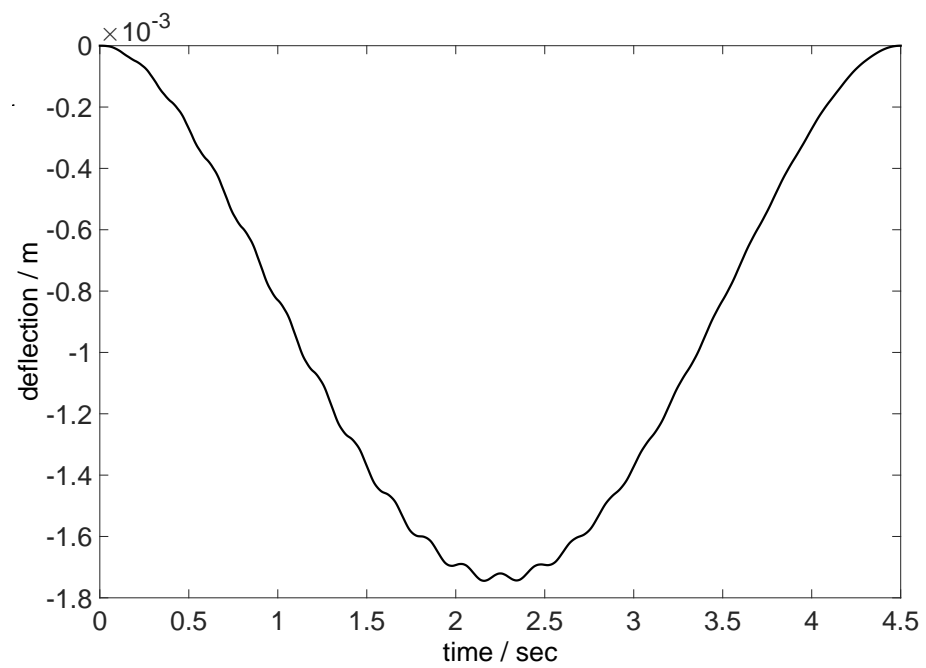

Figure 4.1. The output $y(t)$ of the original model of dimension $N=5000$.

input matrix using the LLS-FEM, LSS-Legendre methods and spline interpolation with $n=30$ combined with model reduction by M-SO-IRKA. We see that for the reduced models of dimension $r=15$, the model reduction errors dominate over the input matrix approximation errors, while for larger dimensions $r$, both types of errors have about the same order. For comparison, we also present in Figures 4.10-4.12 and 4.14-4.16 the absolute errors in the output for the reduced models of different dimensions with the approximated input matrix with $n=50$ and 100 columns. Note that for a fixed dimension $r$, increasing $n$ does not necessarily lead to smaller errors in the output. This can be explained by the fact that the smaller an approximation error in the input matrix is, the larger a model reduction error is, so that the total error remains nearly unchanged.

5. Conclusion. In this paper, we have presented a two-step model reduction approach for mechanical systems subjected to moving loads. Such systems have a time-varying input matrix constructed from the FEM basis functions. The first step consists of an approximation of the time-varying input matrix in a low-dimensional subspace enabling the application of standard model reduction methods to the resulting time-invariant system with a new input in the second step. We have also derived output error bounds which depend on the approximation errors for the input matrix and the model reduction errors. For the approximation of the input matrix, we have used B-spline interpolation and the linear least-squares method with two types of basis functions: Legendre polynomials and FEM basis functions on a coarse grid. For structure-preserving model reduction, we have employed a modified SO-IRKA method. The presented methods have been tested on the Euler-Bernoulli beam model. For such a model, all approximation approaches combined with model reduction give about the same results.

In the future work, it would be interesting to investigate other strategies for the approximation of the input matrix which may depend on time-varying parameters describing the force position. For this purpose, one could use the empirical interpolation method [4] to construct an affine approximation to the non-affine force distribution. 


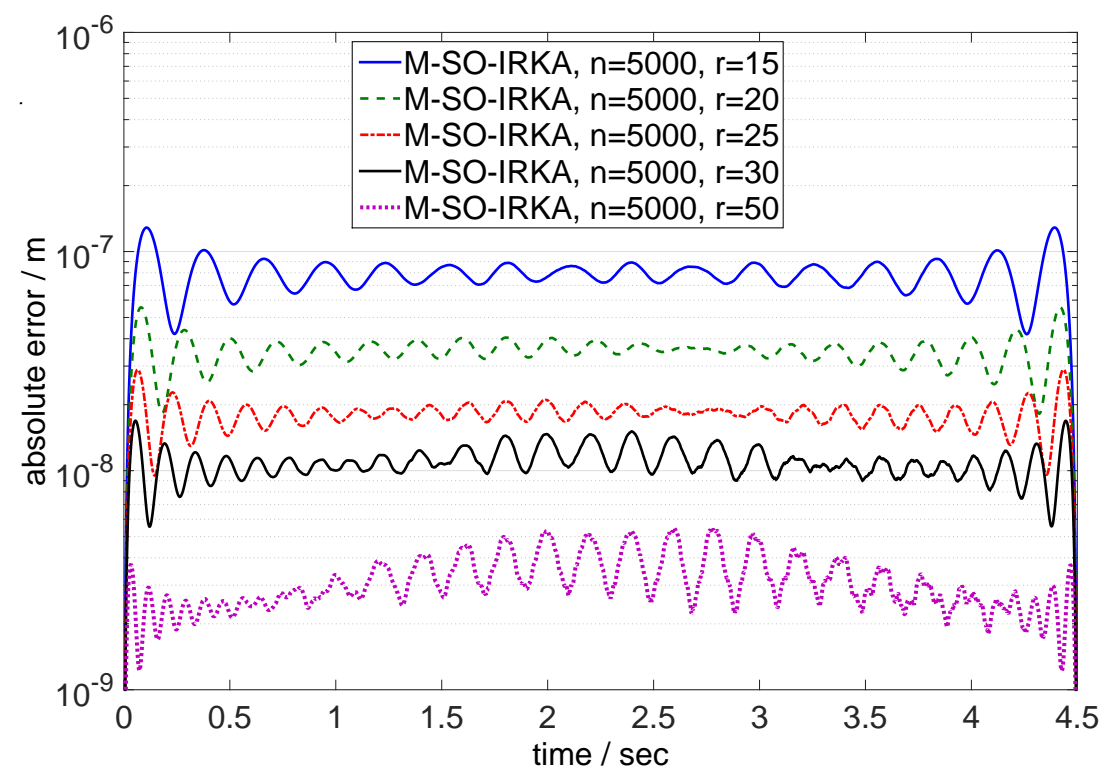

FIGURE 4.2. The absolute errors in the output for the reduced models of different dimensions computed by M-SO-IRKA applied to the system with the input matrix being the identity matrix.

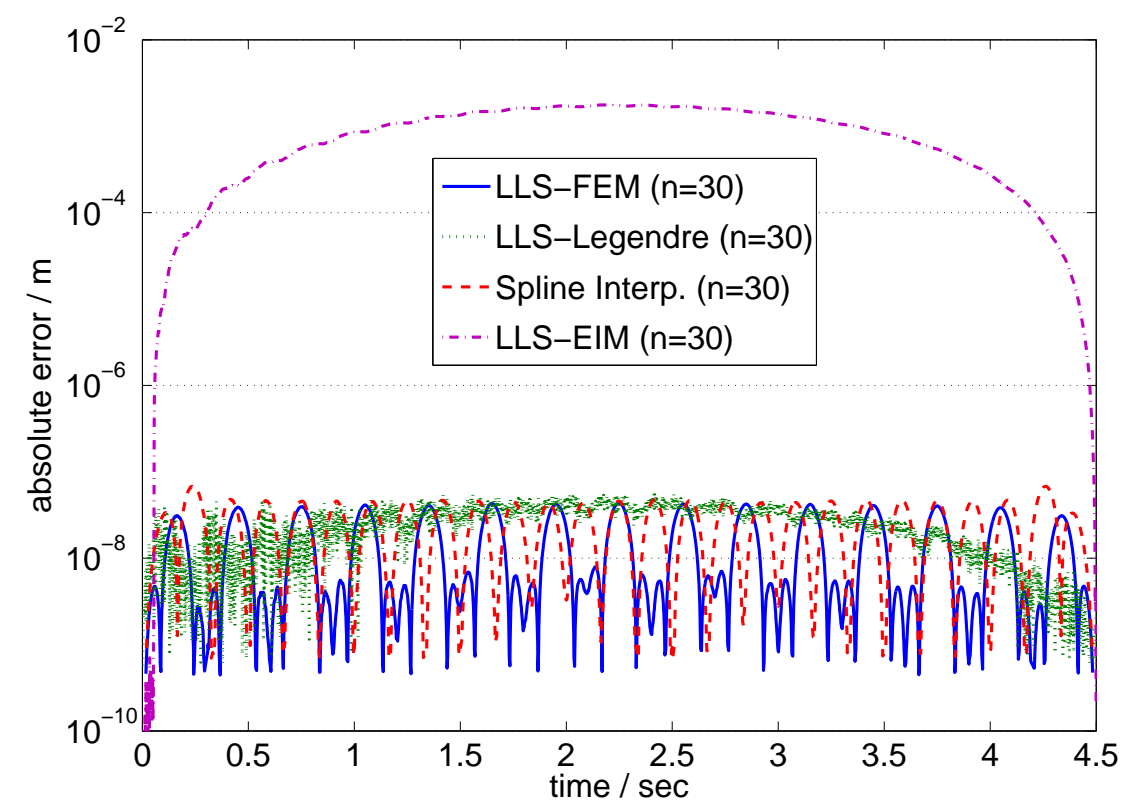

FIGURE 4.3. The absolute errors in the output for the systems with the approximated input matrix $(n=30)$ obtained by four different approaches: LLS-FEM, LLS-Legendre, B-splines interpolation and LLS-EIM. 


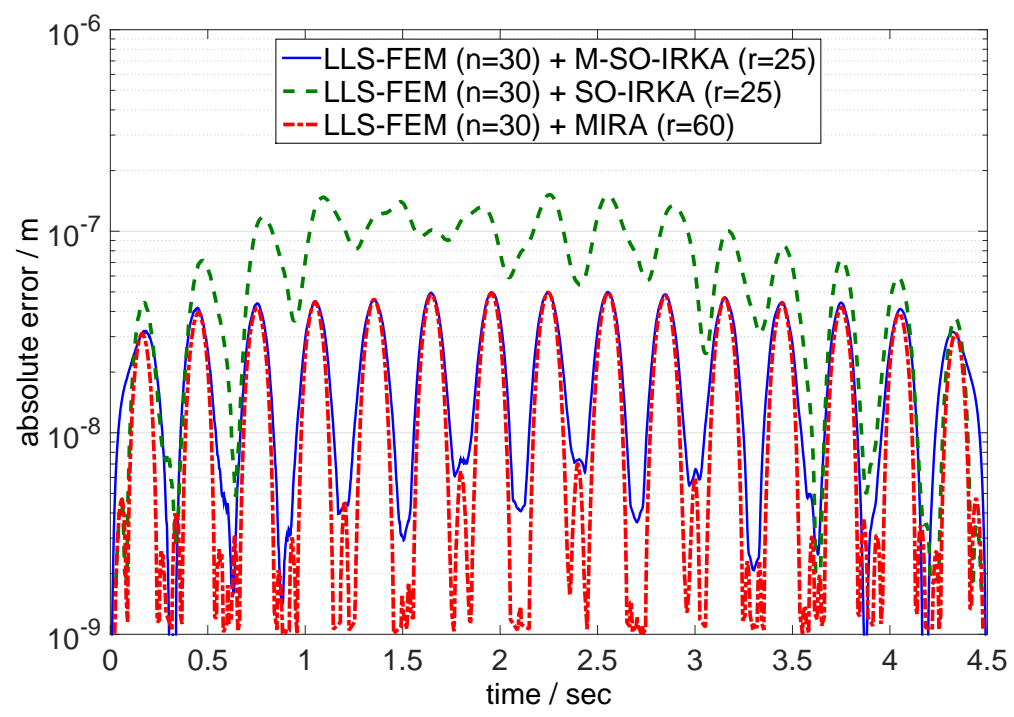

FIGURE 4.4. The absolute errors in the output for the reduced models obtained by the LLS approximation of the input matrix using the FEM basis functions on a coarse grid with $n=30$ combined with model reduction using M-SO-IRKA, SO-IRKA and MIRA.

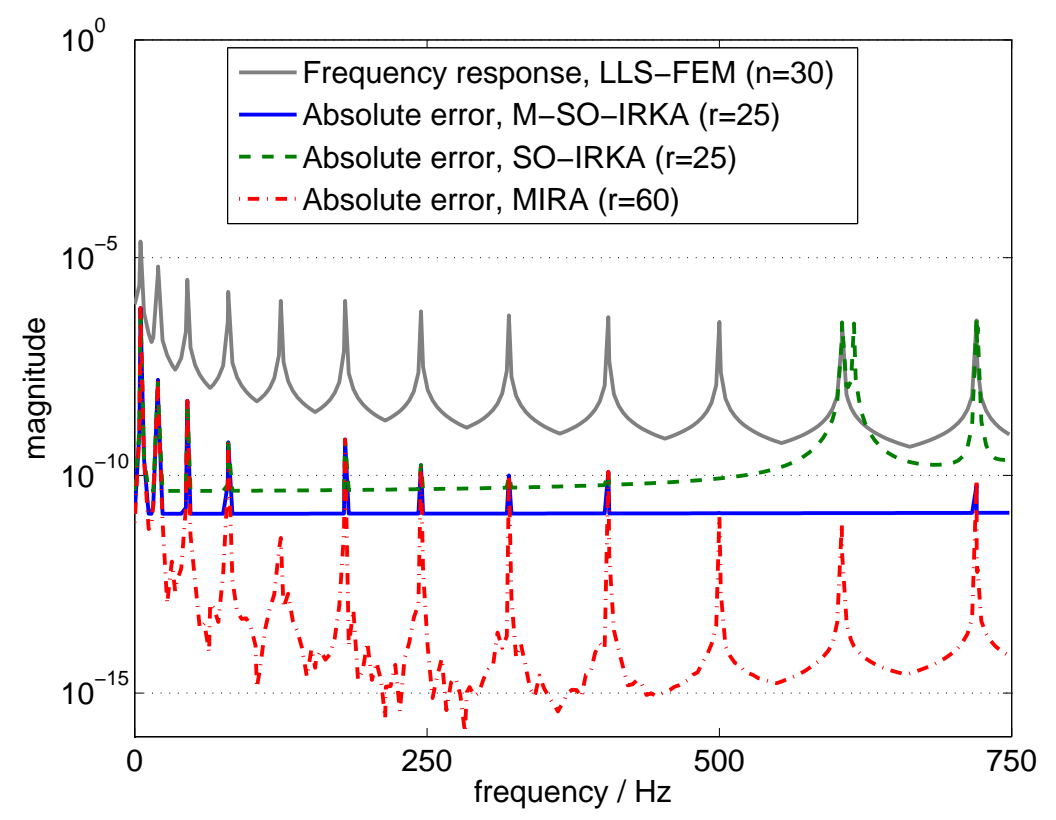

FIGURE 4.5. The spectral norm of the frequency response $\|H(\iota \omega)\|_{2}$ of the system with the approximated input matrix obtained by the LLS approximation using the FEM basis functions on a coarse grid with $n=30$ and the spectral norm of the absolute errors $\|H(\iota \omega)-\widetilde{H}(\iota \omega)\|_{2}$ for the reduced models computed by M-SO-IRKA, SO-IRKA and MIRA. 


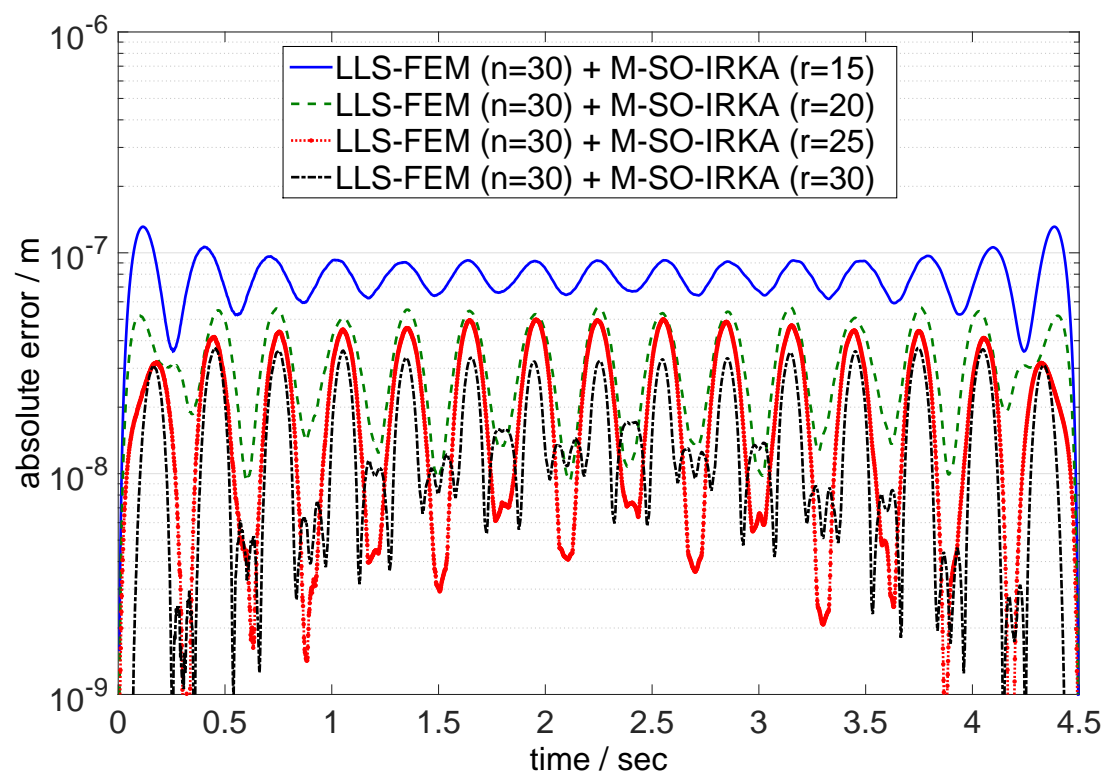

FIGURE 4.6. The absolute errors in the output for the reduced models of different dimensions obtained by the LLS approximation of the input matrix using $n=30$ FEM basis functions on a coarse grid combined with $M-S O-I R K A$.

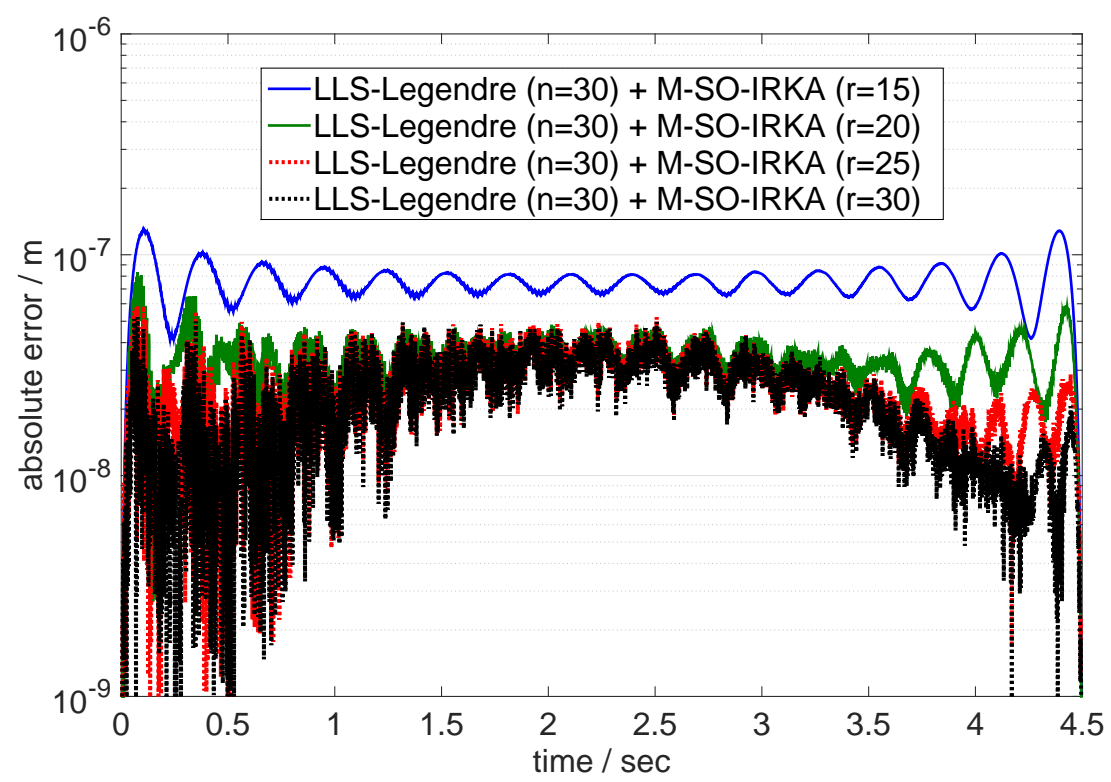

FIGURE 4.7. The absolute errors in the output for the reduced models of different dimensions obtained by the LLS approximation of the input matrix using $n=30$ Legendre polynomials combined with $M-S O-I R K A$. 


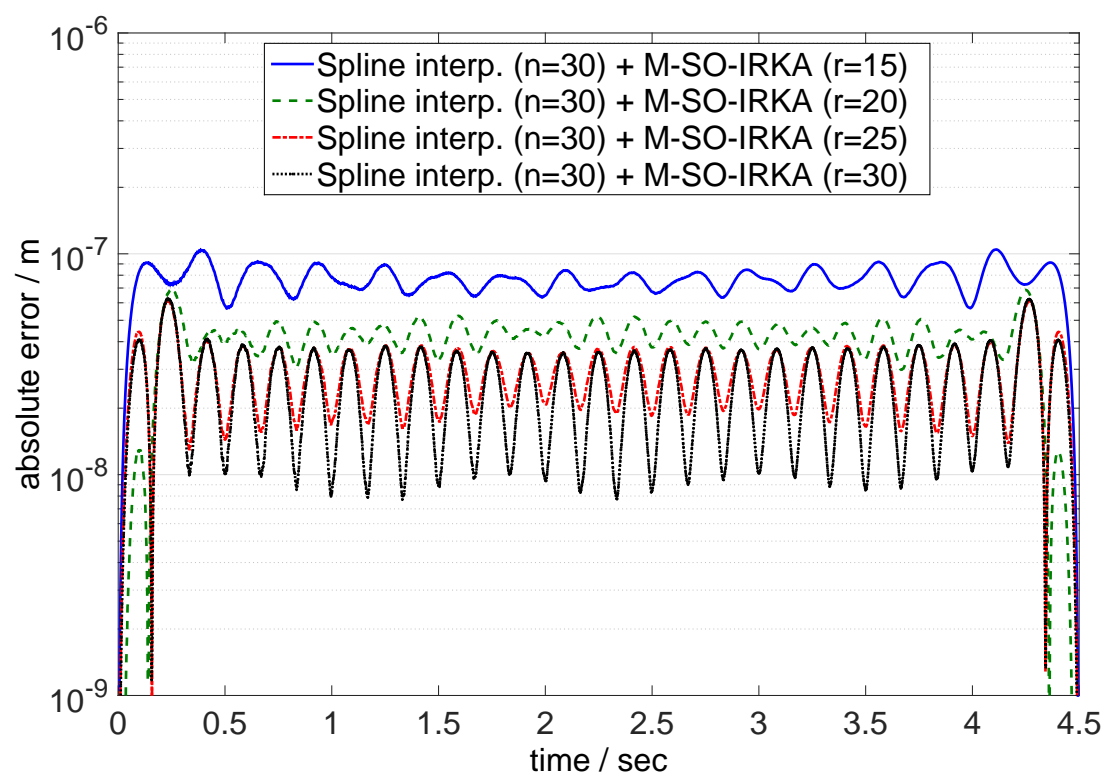

FIGURE 4.8. The absolute errors in the output for the reduced models of different dimensions obtained by the LLS approximation of the input matrix using cubic B-spline interpolation with $n=30$ combined with $M-S O-I R K A$.

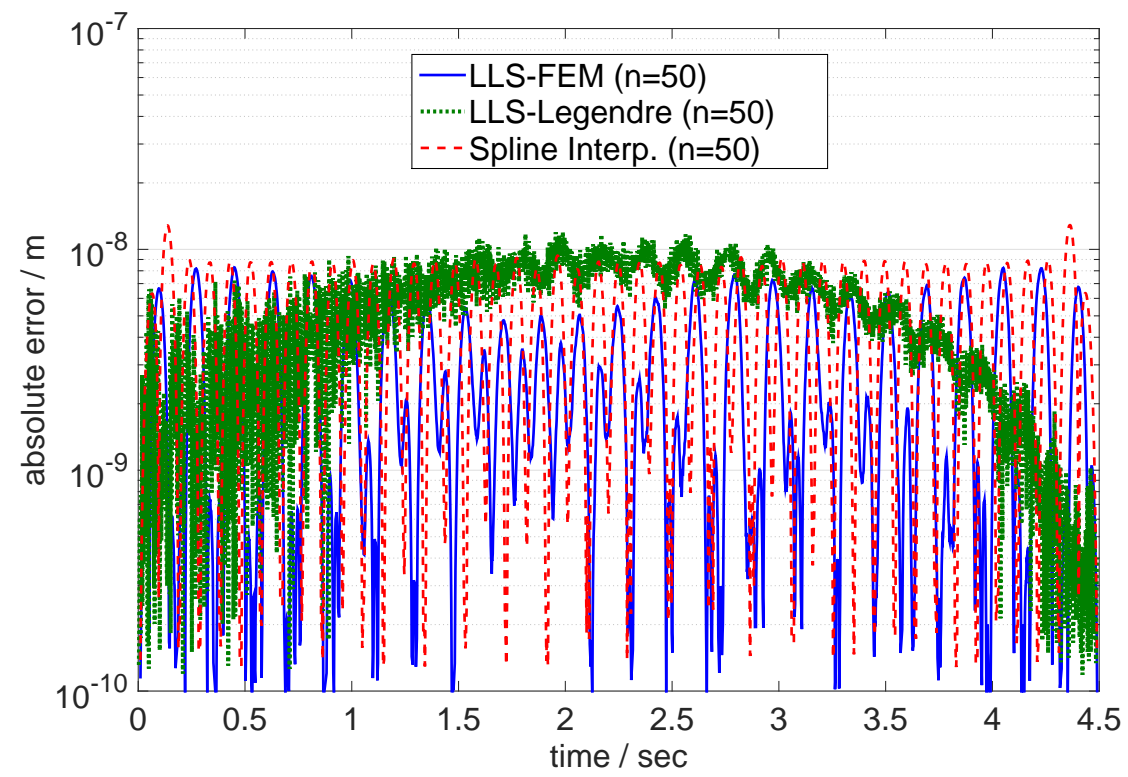

FIGURE 4.9. The absolute errors in the output for the systems with the approximated input matrix $(n=50)$ obtained by three different approaches: LLS-FEM, LLS-Legendre and B-splines interpolation. 


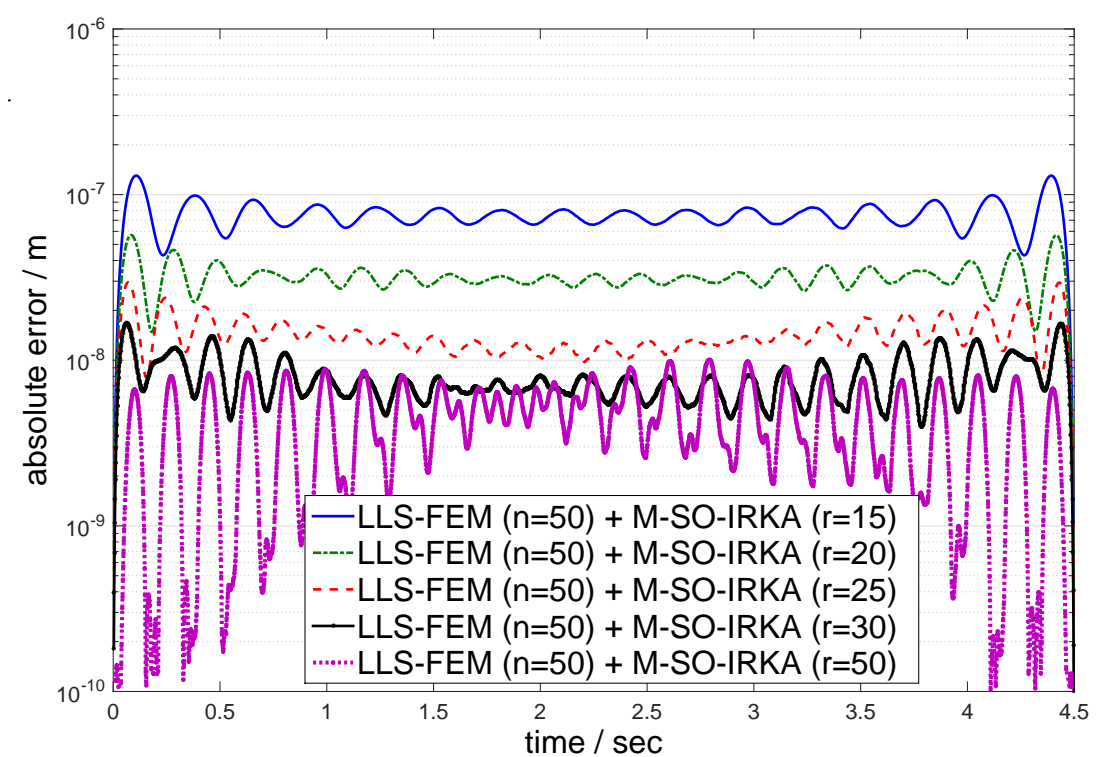

FIGURE 4.10. The absolute errors in the output for the reduced models of different dimensions obtained by the LLS approximation of the input matrix using $n=50$ FEM basis functions on a coarse grid combined with $M-S O-I R K A$.

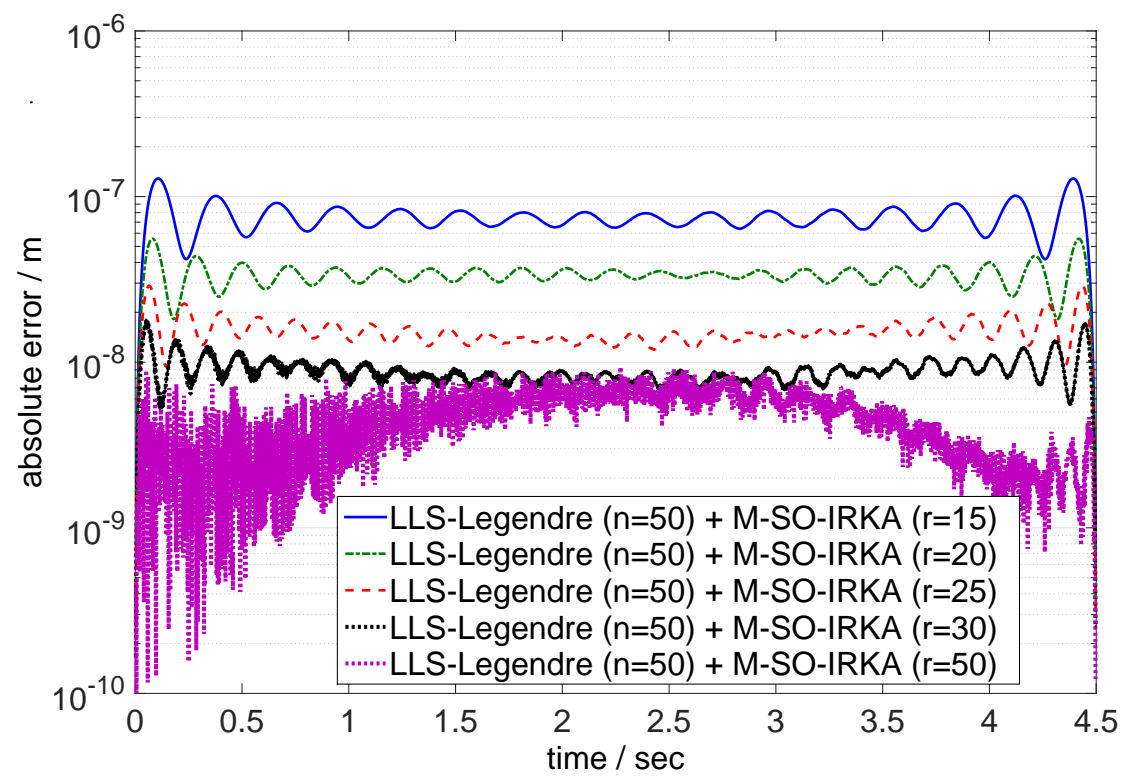

FIGURE 4.11. The absolute errors in the output for the reduced models of different dimensions obtained by the LLS approximation of the input matrix using $n=50$ Legendre polynomials combined with $M-S O-I R K A$. 


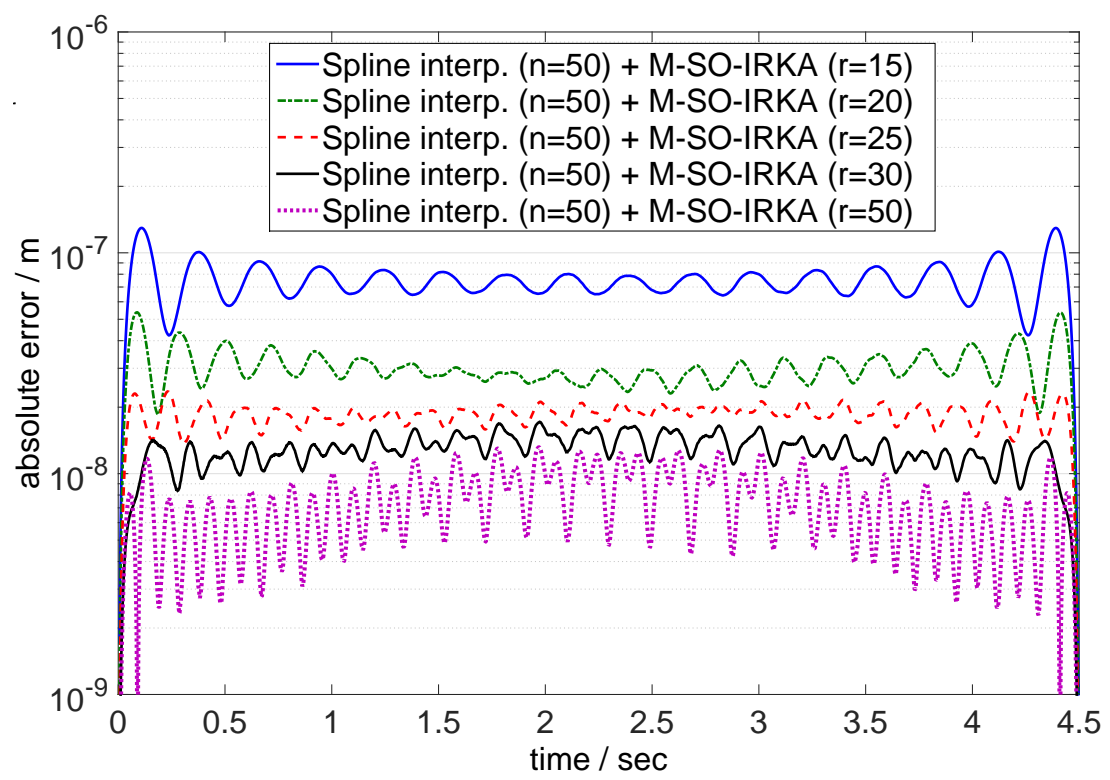

FIGURE 4.12. The absolute errors in the output for the reduced models of different dimensions obtained by the LLS approximation of the input matrix using cubic B-spline interpolation with $n=50$ combined with $M-S O-I R K A$.

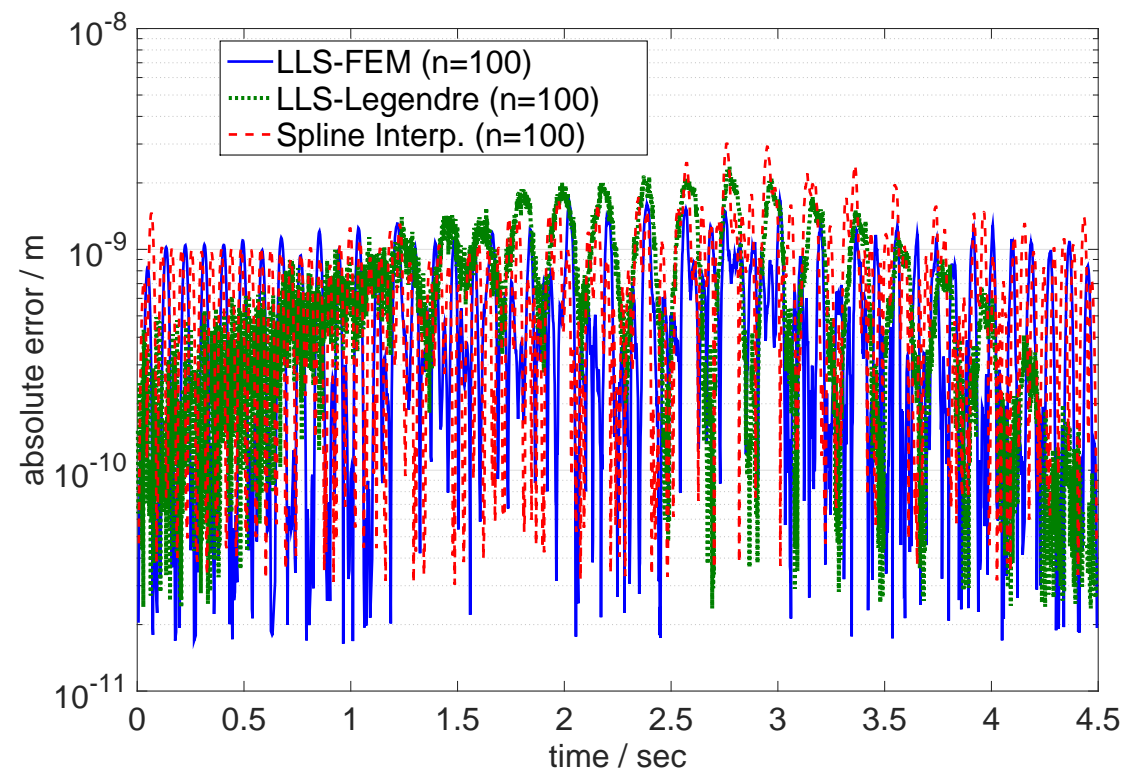

FIGURE 4.13. The absolute errors in the output for the systems with the approximated input matrix $(n=100)$ obtained by three different approaches: LLS-FEM, LLS-Legendre and B-splines interpolation. 


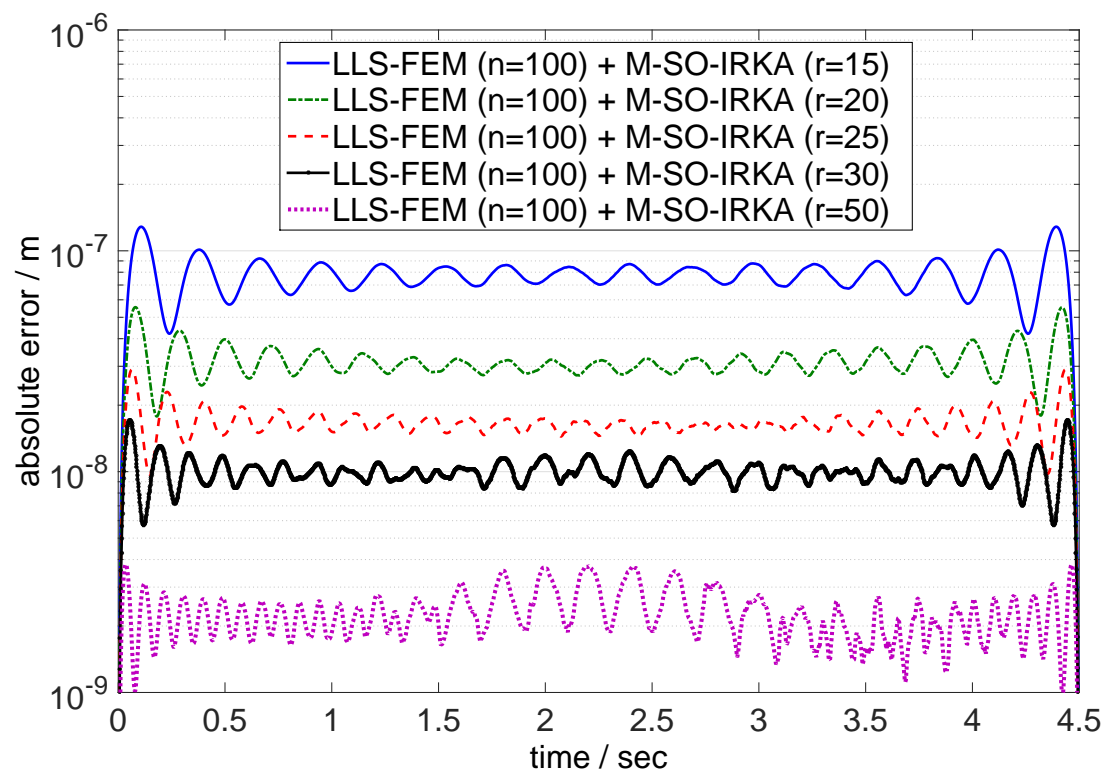

FIGURE 4.14. The absolute errors in the output for the reduced models of different dimensions obtained by the LLS approximation of the input matrix using $n=100$ FEM basis functions on a coarse grid combined with M-SO-IRKA.

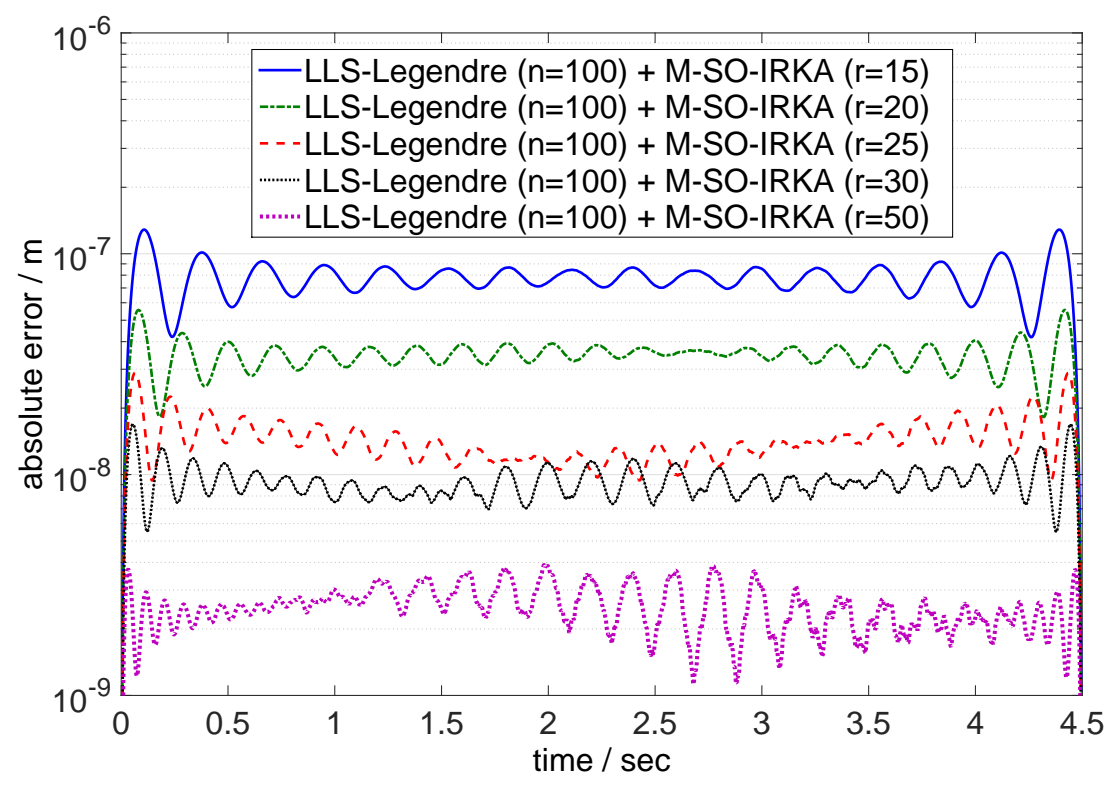

FIGURE 4.15. The absolute errors in the output for the reduced models of different dimensions obtained by the LLS approximation of the input matrix using $n=100$ Legendre polynomials combined with M-SO-IRKA. 


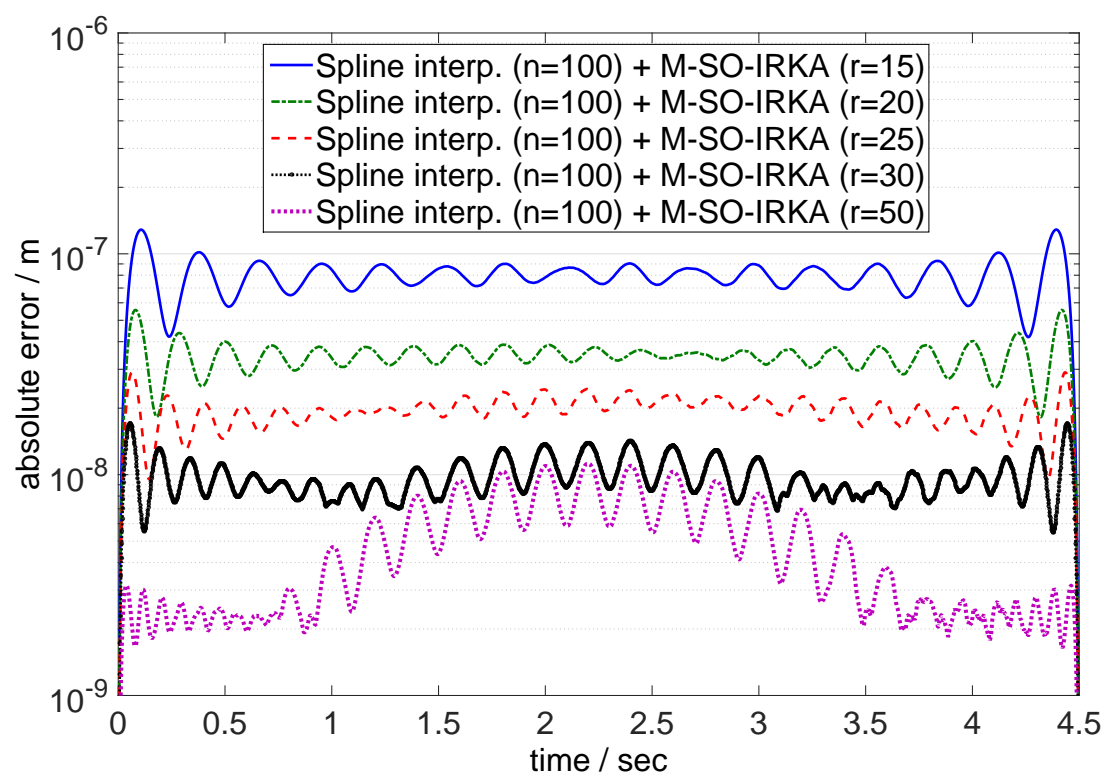

FIGURE 4.16. The absolute errors in the output for the reduced models of different dimensions obtained by the LLS approximation of the input matrix using cubic B-spline interpolation with $n=100$ combined with M-SO-IRKA.

\section{REFERENCES}

[1] A.C. Antoulas, Approximation of Large-Scale Dynamical Systems, SIAM, Philadelphia, PA, 2005.

[2] A.C. Antoulas, C.A Beattie, and S. Gugercin, Interpolatory model reduction of large-scale dynamical systems, in Efficient Modeling and Control of Large-Scale Systems, J. Mohammadpour and K.M. Grigoriadis, eds., Springer-Verlag, New York, 2010, pp. 3-58.

[3] Z. BAI AND Y. Su, Dimension reduction of large-scale second-order dynamical systems via a second-order Arnoldi method, SIAM J. Sci. Comp., 26 (2005), pp. 1692-1709.

[4] M. Barrault, Y. Maday, N.C. Nguyen, and A.T. Patera, An 'empirical interpolation' method: application to efficient reduced-basis discretization of partial differential equations, C.R. Acad. Sci. Paris, 339 (2004), pp. 667-672.

[5] K. Bathe, Finite Element Procedures, Prentice Hall, Upper Saddle River, 1996.

[6] C.A. Beattie And S. Gugercin, Krylov-based model reduction of second-order systems with proportional damping, in Proceedings of the 44th IEEE Conference on Decision and Control (Seville, Spain, December 12-15, 2005), IEEE, 2005, pp. 2278-2283.

[7] E.B. Becker, G.F. Carey, And J.T. Oden, Finite Elements: An Introduction, Prentice-Hall, Inc., Englewood Cliffs, New Jersey, 1981.

[8] P. Benner, P. KÜRSChNer, AND J. SAAK, An improved numerical method for balanced truncation for symmetric second-order systems, Math. Comput. Model. Dyn. Syst., 19 (2013), pp. 593-615.

[9] P. Benner And J. SAAK, Efficient balancing-based MOR for large-scale second-order systems, Math. Comput. Model. Dyn. Syst., 17 (2011), pp. 123-143.

[10] P. Benner And A. Schneider, Model order and terminal reduction approaches via matrix decomposition and low rank approximation, in Scientific Computing in Electrical Engineering SCEE 2008, J. Roos and L.R.J. Costa, eds., vol. 14 of Mathematics in Industry, Springer-Verlag, Berlin, Heidelberg, 2010, pp. 523-530.

[11] G. DAHLQUist, Stability and error bounds in the numerical integration of ordinary differential equations, Almqvist \& Wiksell, Uppsala, 1958.

[12] C. DE Boor, A Practical Guide to Splines, vol. 27 of Applied Mathematical Sciences, SpringerVerlag, New York, 2001. 
[13] P. Feldmann and F. Liu, Sparse and efficient reduced order modeling of linear subcircuits with large number of terminals, in Proceedings of the 2004 IEEE/ACM International Conference on Computer-Aided Design (ICCAD04), Washington, DC, 2004, IEEE Computer Society, pp. 88-92.

[14] M. Fischer and P. Eberhard, Simulation of moving loads in elastic multibody systems with parametric model reduction techniques, Arch. Mech. Engng, 61 (2014), pp. 209-226.

[15] _ . Application of parametric model reduction with matrix interpolation for simulation of moving loads in elastic multibody systems, Adv. Comput. Math., (to appear, DOI 10.1007/s10444-014-9379-7).

[16] R.W. Freund, Padé-type model reduction of second-order systems and higher-order linear dynamical systems, in Dimension Reduction of Large-Scale Systems, P. Benner, V. Mehrmann, and D. Sorensen, eds., vol. 45 of Lecture Notes in Computational Science and Engineering, Springer-Verlag, Berlin, Heidelberg, 2005, pp. 193-226.

[17] L. FrÝBA, Vibration of Solids and Structures under Moving Loads, Thomas Telford Ltd., London, 1999.

[18] E. Grimme, Krylov Projection Methods for Model Reduction, Ph.D. thesis, University of Illinois, Urbana-Champaign, 1997.

[19] S. Gugercin, A.C. Antoulas, and Beattie C., $\mathcal{H}_{2}$ model reduction for large-scale linear dynamical systems, SIAM J. Matrix Anal. Appl., 30 (2008), pp. 609-638.

[20] M.-S. Hossain And P. Benner, Projection based model reduction for time varying descriptor systems using recycled Krylov subspaces, Proceedings in Applied Mathematics and Mechanics, 8 (2008), pp. 10081-10084.

[21] B.I. Kvasov., Shape-Preserving Spline Approximation, World Scientific Publishing Co. Pte. Ltd., Singapore, 2000.

[22] N. Lang, J. SaAk, And P. Benner, Model order reduction for systems with moving loads, at - Automatisierungstechnik, 62 (2014), pp. 512-522.

[23] H.J. Lee, C.C. Chu, And W.S. Feng, An adaptive-order rational Arnoldi method for modelorder reductions of linear time-invariant systems, Linear Algebra Appl., 415 (2006), pp. 235-261.

[24] P. Liu, B. Tan, S.X.-D. Yan, and B. McGaughy, An extended SVD-based terminal and model order reduction algorithm, in Proceedings of the 2006 IEEE International Workshop on Behavioral Modeling and Simulation (San Jose, CA, September 14-15, 2006), IEEE, 2006, pp. 44-49.

[25] D. G. Meyer and S. SRinivasan, Balancing and model reduction for second-order form linear systems, IEEE Trans. Automat. Control, 41 (1996), pp. 1632-1644.

[26] B.C. Moore, Principal component analysis in linear systems: controllability, observability, and model reduction, IEEE Trans. Automat. Control, AC-26 (1981), pp. 17-32.

[27] Y.M. Nechepurenko, Bounds for the matrix exponential based on the Lyapunov equation and limits of the Hausdorff set, Comput. Math. Math. Phys., 42 (2003), pp. 125-134.

[28] B. Osilenker, Fourier Series in Orthogonal Polynomials, World Scientific Publishing Co. Pte. Ltd., Singapore, 1999.

[29] J.R. PhILLIPS, Model reduction of time-varying linear systems using approximate multipoint Krylov-subspace projectors, in Proceedings of the IEEE/ACM International Conference on Computer-Aided Design (ICCAD98, San Jose, CA, USA, November 8-12, 1998), IEEE, 1998, pp. 96-102.

[30] A. Quarteroni, R. Sacco, and F. Saleri, Numerical Mathematics, vol. 37 of Texts in Applied Mathematics, Springer-Verlag, Berlin, Heidelberg, New York, 2000.

[31] T. Reis And T. StYkel, Balanced truncation model reduction of second-order systems, Math. Comput. Model. Dyn. Syst., 14 (2008), pp. 391-406.

[32] J.R. Rieker, Y.-H. Lin, AND M.W. TretheWey, Discretization considerations in moving load finite element beam models, Finite Elem. Anal., 21 (1996), pp. 129-144.

[33] B. Salimbahrami And B. Lohmann, Order reduction of large scale second-order systems using Krylov subspace methods, Linear Algebra Appl., 415 (2006), pp. 385-405.

[34] H. SAndberg And A. RAntzer, Balanced truncation of linear time-varying systems, IEEE Trans. Automat. Control, 49 (2004), pp. 217-229.

[35] W.H.A. Schilders, H.A. van Der Vorst, and Rommes J., eds., Model Order Reduction: Theory, Research Aspects and Applications, vol. 13 of Mathematics in Industry, SpringerVerlag, Berlin, Heidelberg, 2008.

[36] S. Shokoohi, L.M. Silverman, and P.M. Van Dooren, Linear time-variable systems: Balancing and model reduction, IEEE Trans. Automat. Control, 28 (1983), pp. 810-822.

[37] A. SoppA, Krylov-Unterraum basierte Modellreduktion zur Simulation von Werkzeugmaschinen, Ph.D. thesis, Technische Universität Braunschweig, 2011. 
[38] K. Veselić, Bounds for exponentially stable semigroups, Linear Algebra Appl., 358 (2003), pp. 309-333.

[39] J. WANG AND I. Howard, Finite element analysis of high contact ratio spur gears in mesh, J. Tribology, 127 (2005), pp. 469-483.

[40] S. WyatT, Issues in Interpolatory Model Reduction: Inexact Solves, Second-order Systems and $D A E s$, Ph.D. thesis, Virginia Polytechnic Institute and State University, 2012.

[41] O.C. Zienkiewicz, R.L. Taylor, And D. Fox, eds., The Finite Element Method for Solid and Structural Mechanics, Butterworth-Heinemann, Oxford, 2014.

[42] N.Đ. ZRnić, V.M. Gašść, S.M. BošnJak, AND M. Đordević, Moving loads in structural dynamics of cranes: bridging the gap between theoretical and practical researches, in Proceedings of the 11-th International Conference on Vibration Problems (ICOVP 2013, Lisbon, Portugal, September 9-12, 2013), paper 79_0, 2013. 